\title{
ARTICLE Modulating microglia activation prevents maternal immune activation induced schizophrenia-relevant behavior phenotypes via arginase 1 in the dentate gyrus
}

\author{
Yucen Xia ${ }^{1}$, Zhiqing Zhang ${ }^{1}$, Weipeng $\mathrm{Lin}^{2}$, Jinglan Yan ${ }^{1}$, Chuan'an Zhu ${ }^{1}$, Dongmin Yin iD $^{2}$, Su He ${ }^{1}$, Yang Su ${ }^{1}$, Nenggui Xu ${ }^{1}$, \\ Robert William Caldwell ${ }^{3}$, Lin Yao ${ }^{1,4}$ and Yongjun Chen ${ }^{1,5,6}$
}

Prenatal infection during pregnancy increases the risk for developing neuropsychiatric disorders such as schizophrenia. This is linked to an inflammatory microglial phenotype in the offspring induced by maternal immune activation (MIA). Microglia are crucial for brain development and maintenance of neuronal niches, however, whether and how their activation is involved in the regulation of neurodevelopment remains unclear. Here, we used a MIA rodent model in which polyinosinic: polycytidylic acid (poly $(\mathrm{I}: \mathrm{C}))$ was injected into pregnant mice. We found fewer parvalbumin positive (PV+) cells and impaired GABAergic transmission in the dentate gyrus (DG), accompanied by schizophrenia-like behavior in the adult offspring. Minocycline, a potent inhibitor of microglia activation, successfully prevented the above-mentioned deficits in the offspring. Furthermore, by using microglia-specific arginase 1 (Arg1) ablation as well as overexpression in DG, we identified a critical role of Arg1 in microglia activation to protect against poly $(\mathrm{I}: \mathrm{C})$ imparted neuropathology and altered behavior in offspring. Taken together, our results highlight that Arg1mediated alternative activation of microglia are potential therapeutic targets for psychiatric disorders induced by MIA.

Neuropsychopharmacology (2020) 45:1896-1908; https://doi.org/10.1038/s41386-020-0743-7

\section{INTRODUCTION}

Epidemiological studies implicate a correlation between prenatal virus infection and an increased risk in the development of postnatal psychiatric disorders including schizophrenia and autism $[1,2]$. Accumulating evidence in the field of neurodevelopment supports the idea that prenatal exposure to viruses or immune challenges is a key pathological factor, resulting in schizophreniarelevant phenotypes in the offspring, from juvenile to adult stages of life $[3,4]$. Studies in animal models have identified that maternal immune activation (MIA) alone is sufficient to induce fetal brain developmental abnormalities and behavior deficits in later life [5]. As one of the most commonly used MIA models, polyinosinic: polycytidylic acid (poly $(\mathrm{I}: \mathrm{C})$ ) intervention in rodents resulted in pathological brain changes in the offspring, including deficits in long-range connections between brain regions, decreased GABAergic input, as well as altered synaptic plasticity and function $[6,7]$. Some studies reveal that exposure to poly $(I: C)$ during pregnancy results in an increase of pro-inflammatory cytokines and activated microglia in the brain, which are considered the main reasons for neuropathogenesis in the progeny $[8,9]$.

Microglia, the resident immune cells of the brain, are key to adequate development of the central nervous system (CNS) and play functions directly in various brain pathologies $[10,11]$. It is worth noting that the postmortem analysis of schizophrenic patients shows increased microglia cellular density and activity in multiple brain regions [12]. Furthermore, numerous studies have observed microglia inflammatory activation, as well as an increased number of inflammatory cells, in a variety of animal models of psychiatric disorders [2, 13]. It has also been confirmed that the response of microglia to pathological changes in the brain leads to dysfunction of the neurotrophic system together with the release of inflammatory cytokines [14, 15].

Recent studies on whether MIA can induce alterations of microglia activity are contentious. Multiple studies have reported the obvious deficiency in microglia density, the expression of microglia activation markers as well as the morphological appearance of microglia in MIA rodent models $[16,17]$. However, other studies show no obvious changes in microglia activity in the MIA offspring $[18,19]$. While it is possible that differences in the involved brain region and the time point when microglia are analyzed among these studies may contribute to the contradictory results [11], it has been proposed that MIA could lead to microglia activation, resulting in the abnormal behavioral phenotype and neuronal deficits $[2,20]$. Thus, more evidence is needed to support this hypothesis.

Anti-inflammatory intervention can inhibit microglial activation and treat neuropsychiatric disorders [21]. As a semisynthetic second-generation tetracycline, minocycline is a clinically effective

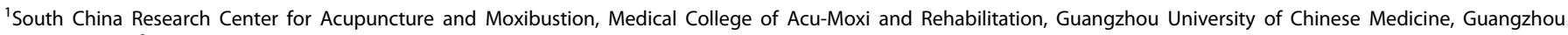

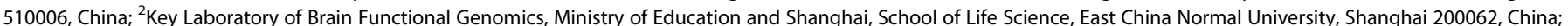

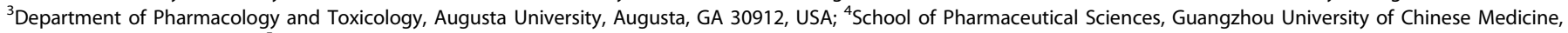

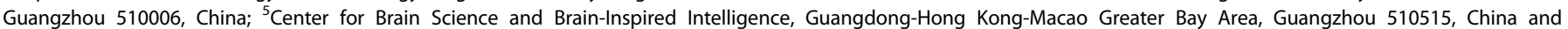
${ }^{6}$ Guangdong Province Key Laboratory of Psychiatric Disorders, Southern Medical University, Guangzhou 510515, China Correspondence: Lin Yao (lyao526@gzucm.edu.cn) or Yongjun Chen (ychen@gzucm.edu.cn)
}

Received: 7 January 2020 Revised: 10 June 2020 Accepted: 16 June 2020

Published online: 29 June 2020 
anti-inflammatory drug, which has been shown to delay the incidence of schizophrenia [22, 23]. In animal models of schizophrenia, two weeks of minocycline administration alleviates hyperactivity, sensory gating, and cognitive behavior deficits, and inhibits microglia activation in the offspring exposed to MIA $[24,25]$. However, the critical molecular signaling that regulates microglial activation remains unknown.

As an alternative microglial activation marker, arginase 1 (Arg1) competes against inducible nitric oxide synthase for L-arginine and inhibits the production of nitric oxide (NO) $[26,27]$. The abnormal expression of microglia Arg1 has been reported in CNS injury. Increased Arg1 expression is involved in the suppression of neuroinflammation in traumatic spinal cord injury, experimental autoimmune encephalomyelitis and acute stroke [28, 29]. In an Alzheimer's disease mouse model, Arg1+ microglia were induced by IL-4 injection, which significantly reduced $A \beta$ plaque deposition during IL-1 $\beta$ dependent neuroinflammation [30]. Furthermore, the number of Arg1+ microglia in close proximity to motor neurons was increased during amyotrophic lateral sclerosis progression [31]. In addition, the alternative microglia phenotype that accompanies changes of Arg1 expression improved cognitive impairment induced by maternal sleep deprivation [32], suggesting Arg1 may be a key target for regulating neuronal inflammation. Therefore, it is necessary to investigate the critical role of microglia Arg1 in MIA-induced neuropathogenesis.

In the current study, we explored new pathological changes regulated by microglia activation, which are related to MIAinduced schizophrenia-like behavior in the offspring. Furthermore, we confirmed that Arg1 is a key factor in the regulation of microglia activation and is involved in MIA-induced pathological processes during brain development.

\section{MATERIALS AND METHODS}

A brief summary of experimental procedures is provided here with additional details available in the Supplementary Information (SI) accompanying the article. All animal procedures were approved by the Animals Care and Use Committee of Guangzhou University of Chinese Medicine.

Animals

Adult male and female C57BL/6 mice were purchased from the Laboratory Animal Center of Sun Yat-sen University. The females and males mated at a ratio of 2 to 1 . The presence of a vaginal plug was defined as gestational day 0 (GD0). Floxed Arg1 mice were obtained from Dr. Caldwell (Augusta University, Augusta, USA). as described in our previous studies $[33,34]$ and CX3CR1CreER mice were a gift from Dr. Yin (East China Normal University, Shanghai, Stock No: 021160, Jackson Laboratory). The mice were housed in an Individual Ventilated Caging System (IVC, Allentown Inc.) with a 12-h light/dark cycle (lights on at 7:00 A.M., humidity $60 \pm 5 \%$, temperature $24 \pm 1{ }^{\circ} \mathrm{C}$ ) and ad libitum food and water. The offspring mice were caged (3-4 mice per cage) with same-sex littermates after postnatal day 21 (PD21). Unless otherwise noted, all test were conducted with male offspring.

\section{Poly $(\mathrm{I}: \mathrm{C})$ administration}

Poly $(I: C)$ administration was carried out as described previously $[35,36]$. Poly (I:C) (potassium salt; Sigma-Aldrich, \#P9582) was dissolved in sterile pyrogen-free $0.9 \% \mathrm{NaCl}$ to yield a final concentration of $2 \mathrm{mg} / \mathrm{ml}$ ). Pregnant mice received either an intravenous injection of Poly $(\mathrm{l}: \mathrm{C})(5 \mathrm{mg} / \mathrm{kg})$ or vehicle (sterile pyrogen-free $0.9 \% \mathrm{NaCl}$ ) on $\mathrm{GD} 12$.

\section{Minocycline administration}

Minocycline administration was carried out as described previously [24]. Briefly, minocycline hydrochloride $(40 \mathrm{mg} / \mathrm{kg}$, SigmaAldrich, \#M9511) was dissolved in sterile pyrogen-free $0.9 \% \mathrm{NaCl}$ to yield a final concentration of $10 \mathrm{mg} / \mathrm{ml}$. The offspring were given an intraperitoneal injection of minocycline hydrochloride (40 mg/kg, once daily) or vehicle (sterile pyrogen-free $0.9 \% \mathrm{NaCl}$ ) from PD21 for 2 weeks. Mice were randomly divided into the following groups: offspring from pregnant mice of saline-injection that received vehicle (Ctrl + vehicle); offspring from pregnant mice of saline-injection that received minocycline (Ctrl + Mino); offspring from pregnant mice of poly $(\mathrm{I}: \mathrm{C})$ injection that received vehicle (Poly+vehicle); offspring from pregnant mice of poly $(I: C)$ injection that received minocycline (Poly + Mino).

\section{Tamoxifen administration}

Tamoxifen administration was carried out as described previously [37]. Tamoxifen (TAM, Sigma-Aldrich, \#T5648) was dissolved in corn oil at $20 \mathrm{mg} / \mathrm{ml}$. To induce CreER activity, CX3CR1-CreER or CX3CR1-Arg1 $1^{\mathrm{f} / \mathrm{f}}$ mice were administered intraperitoneal TAM $(100 \mathrm{mg} / \mathrm{kg}$, once a day over 7 days) from PD21. As the control of CX3CR1-Arg $1^{\mathrm{f} / \mathrm{f}}$ mice, littermates $\left(\operatorname{Arg} 1^{\mathrm{f} / \mathrm{f}}\right.$ ) also received the TAM administration.

\section{Genotyping primers}

All transgene mouse lines used in this study were confirmed by genotyping analysis with PCR. Genotyping primer sequences were as follows: Floxed Arg 1, 5'-TGC GAG TTC ATG ACT AAG GTT-3' and 5'-AAA GCT CAG GTG AAT CGG-3'; CX3CR1-CreER, 5'-AAG ACT CAC GTG GAC CTG CT-3', 5'-CGG TTA TTC AAC TTG CAC CA-3' and 5'-AGG ATG TTG ACT TCC GAG TTG-3'.

\section{Behavioral tests}

The behavioral tests started at PD56 and were performed by a person blind to the experimental groups and conducted based on our previous protocols [38]. The offspring mice were tested on the open field, prepulse inhibition (PPI), social withdrawal, and Morris water maze (MWM) task. For details, see SI Methods. Both male and female offspring were used in behavior studies separately.

Immunohistochemistry (IHC)

Sections were processed for staining using our previously described immunofluorescence procedure [39]. Mice were anesthetized and transcardially perfused with $4 \%$ paraformaldehyde (PFA). Free-floating, 40- $\mu \mathrm{m}$-thick coronal sections of the entire hippocampus were collected on a freezing microtome (Leica SM2010R). The primary antibodies were as follows: rabbit anti-PV (1:500; Abcam), chicken anti-Arg1 (1:1000; from Dr. Robert W. Caldwell, Augusta University, USA), rabbit anti-lba1 (1:500; Abcam) and mouse anti-GFAP (1:1000 Sigma). The secondary antibodies include Alexa Fluor 488 goat anti-rabbit (1:1000; Abcam), FITC goat anti-chicken (1:1000; Invitrogen), Alexa Fluor 594 goat anti-rabbit (1:1000; Abcam) and Alexa Fluor 594 goat anti-mouse (1:1000; Abcam). For details, see SI Methods.

\section{Confocal and MBF stereo-investigation}

For microscopic analysis, we used a Nikon A1 confocal laser scanning microscope. Cell number was assessed by unilateral counting of parvalbumin positive $(\mathrm{PV}+$ ) cells in the hippocampus by the optical-fractionator method with a stereology system stereo investigator (MBF Bioscience, Williston, VT, USA). Actual section thickness was measured, and appropriate guard zones at the top and bottom of the section were defined to avoid oversampling. Measurements were made in an equidistant series of six coronal sections $(240 \mu \mathrm{m}$ apart $)$ with a starting point at $\mathrm{A} / \mathrm{P}-1.46 \mathrm{~mm}$ from bregma and spanning the entire hippocampus in its rostrocaudal extension.

Microglial cell density and ramification index analyses Microglial cell density and ramification index analyses were performed as described previously [40]. Briefly, quantification of the number of microglia cells per $\mathrm{mm}^{2}$ and their average total 
process length per cell $(\mu \mathrm{m})$ were performed using ImageJ software (NIH, Bethesda, Maryland, USA). Iba1 positive cells were counted manually and analyzed live at $\times 20$ magnification. The following sampling parameters were used: (1) a fixed counting frame of $200 \times 200 \mu \mathrm{m}$, (2) a sampling grid size of $1000 \times 600 \mu \mathrm{m}$. The counting frames were placed randomly at the intersections of the grid within the outlined structure of interest by the software. For ramification analysis, the maximum intensity projection of the Iba1 positive signal was used in order to fully visualize all the microglia cell processes. All coronal slices were quantified in the entire hippocampus with reference to bregma $(-1.46$ to -2.66 $\mathrm{mm}$ ). Estimations were carried out in every 6th section, with six sections per animal counted.

\section{Western blot}

Western blot analysis was performed as previously described [38]. For details, see SI Methods. Briefly, mice were sacrificed by rapid decapitation under deep anesthesia. The brain tissues were dissected in ice-cold 1X PBS buffer, then the samples were homogenized and centrifuged. Total protein concentration was determined using an Enhanced BCA Protein Assay Kit (Beyotime). The following antibodies were used: rabbit anti-PV antibody (1:4000, Abcam), rabbit anti-Arg1 antibody (1:5000, Abcam), rabbit anti- $\beta$-tubulin antibody (1:5000, Cell Signaling Technology), rabbit anti-IL1 $\beta$ antibody (1:2000, Abcam), rabbit anti-IL6 antibody (1:3000, GeneTex), rabbit anti-TNFa antibody (1:3000, GeneTex), and goat anti-rabbit IgG $(\mathrm{H}+\mathrm{L})$ HRP (1:8000, Abways). Protein bands were visualized using a chemiluminescence system (ChemiDocTM XRS+, BioRad). The protein expressions were semi-quantitatively analyzed with ImageJ software.

\section{Slice preparation and electrophysiological recordings}

The acute hippocampal brain slices in electrophysiology experiments were prepared as previously described [41]. Briefly, mice were anesthetized with $1.25 \%$ avertin $(0.2 \mathrm{ml} / 10 \mathrm{~g}$, Sigma, USA) and decapitated. Brains were quickly removed and chilled in icecold artificial cerebrospinal fluid (ACSF) bubbled with a mixture of $5 \% \mathrm{CO}_{2}$ and $95 \% \mathrm{O}_{2}$. Hippocampal slices $(300 \mu \mathrm{m})$ were prepared using a Vibroslice (VT 1200S; Leica). All slices were recovered for $30 \mathrm{~min}$ at $32{ }^{\circ} \mathrm{C}$ followed by $1 \mathrm{~h}$ at room temperature in ACSF bubbled with a mixture of $5 \% \mathrm{CO}_{2}$ and $95 \% \mathrm{O}_{2}$. For miniature inhibitory postsynaptic current (mIPSC) recording, whole-cell voltage clamp recordings of CA1 pyramidal neurons or granular cells (GCs) in DG were aided with infrared optics using an upright microscope equipped with a $40 \mathrm{x}$ water-immersion lens (Nikon, FN1, Japan) and an infrared-sensitive charge-coupled device (CCD) camera (C11440, HAMAMATSU, Japan). All recordings were made with a Multiclamp 700B amplifier and 1550B digitizer (Axon Instrument, Molecular Devices). For details, see SI Methods.

Stereotaxic adeno-associated virus (AAV) injection

The Arg1 overexpression AAV (pAAV-CMV-DIO-Arg1-eGFP-2A, $9.38 \times 10^{12} \mathrm{VG} / \mathrm{ml}$ ), and control AAV for Arg1 overexpression AAV $\left(2.31 \times 10^{12} \mathrm{VG} / \mathrm{ml}\right)$, with a serotype of AAV9, were purchased from Sunbio Medical Biotechnology (Shanghai, China). Mice were fixed on a stereotactic frame (RWD Life Science, Shenzhen, China). The virus was injected through a glass microelectrode using the GLASS REPLACEMENT 3.5 NANOLTR (World Precision Instrument, Item NO. 4878) while mice were under anesthesia with pentobarbital sodium solution. Injections were bilaterally made at $A / P-2.0 \mathrm{~mm}$, $\mathrm{M} / \mathrm{L} \pm 1.5 \mathrm{~mm}$, and $\mathrm{D} / \mathrm{V}-1.8 \mathrm{~mm}$ from bregma with a dose of $1 \mu \mathrm{l}$ $\left(9.38 \times 10^{12} \mathrm{VG} / \mathrm{ml}\right)$ on each side. CX3CR1-creER Mice recovered for 5 weeks after virus injection before experiments to allow sufficient expression of the transgene.

Microglia isolation and real-time PCR

Microglia isolation and mRNA quantification by real-time PCR were performed as previously described [42]. Briefly, we isolated microglial cells with $40-70 \%$ Percoll followed by fluorescenceactivated cell sorter, then the mRNA level of Arg1 was further detected by qRT-PCR. For details, see SI Methods.

Statistical analysis

Data were analyzed using SPSS version 21.0 (SPSS, Inc., Chicago, IL, USA). Statistical analysis of the data were performed using either one-way, two-way or three-way ANOVA followed by Bonferroni post-hoc tests. Two-way or three-way ANOVA with repeated measures were used to analyze the results of the escape latency in the Morris and PPI tasks. All data were presented as the mean \pm SEM, and $p<0.05$ was considered statistically significant.

\section{RESULTS}

Minocycline administration in juvenile period ameliorates schizophrenia-like behavior of adult poly $(\mathrm{I}: \mathrm{C})$ offspring Progeny exposed to prenatal immune challenges is mainly characterized by schizophrenia-relevant behavior in terms of hyperactivity, startle gating, social withdrawal, and cognitive function, which are similar to positive, negative, and cognitive symptoms displayed by human schizophrenic patients [43, 44]. Furthermore, our previous experiments found that the number of $\mathrm{PV}+$ interneurons in DG was decreased at PD35 in poly $(\mathrm{I}: \mathrm{C})$ offspring, but not at PD21 (data not shown). To determine whether minocycline administration in the juvenile period can prevent MIA-induced psychiatric disorders, male offspring mice were administered minocycline from PD21 for 2 weeks and behavior tests were performed on PD56 (Fig. 1a). First, we detected locomotor activity using an open field test. Our results showed that Poly+vehicle offspring were hyperactive compared with $\mathrm{Ctrl}+$ vehicle offspring, as demonstrated by an increase of the total distance traveled in the open field increased. Nevertheless, minocycline treatment attenuated MIA-induced hyperactivity (Fig. 1b).

We next performed PPI test to assess deficiencies in sensorymotor gating. All four groups showed a similar response to $70 \mathrm{~dB}$ background noise suggesting no deficit in hearing (Fig. 1c). Compared with Ctrl+vehicle offspring, PPI was impaired in Poly +vehicle offspring, which was ameliorated by the minocycline treatment (Fig. 1d). Next, we investigated the effects of minocycline on social interaction using the three-chamber test. No significant differences were found between groups regarding the time spent around the cylinder without a stimulus mouse inside. In the presence of a stimulus mouse, the time spent on this stimulus was significantly reduced in Poly+vehicle offspring compared with $\mathrm{Ctrl}+$ vehicle offspring. However, minocycline administration failed to alleviate this social deficit (Fig. 1e).

We further determined the spatial memory in the MWM task. During a 4-day training session, the latency to find the hidden platform was upregulated in Poly+vehicle offspring, compared to Ctrl+vehicle offspring, indicating an inability of Poly+vehicle offspring to learn. Poly+Mino offspring had an obviously shorter latency than Poly+vehicle offspring (Fig. 1g). Subsequently, we carried out a probe trial to measure the memory retention of the mice to the hidden platform location on day 5. The Poly+vehicle offspring spent significantly less time in the target quadrant than Ctrl+vehicle offspring, indicating an impaired spatial memory. Poly+Mino offspring spent more time in the target quadrant than Poly+vehicle offspring (Fig. 1h). The above results demonstrate that minocycline treatment during the juvenile period alleviated behavior deficits in locomotor activity, sensory gating, and cognitive ability induced by prenatal poly (I:C) insult, but not social withdrawal in male offspring. Considering the possibility of the sex-specific effects in animal behaviors of MIA, the female offspring were also used in behavior tests [45-47]. We found that the female mice exposed to MIA have similar behavior deficits as male mice, and minocycline treatment can prevent these behavior 
A

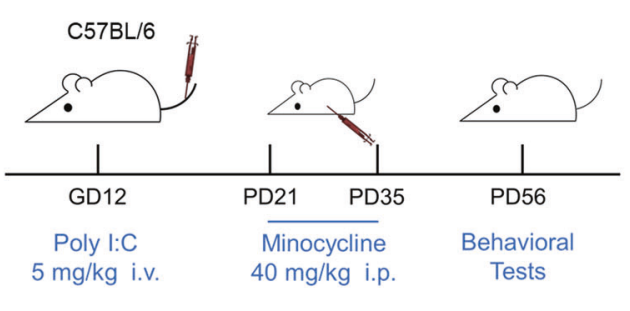

D

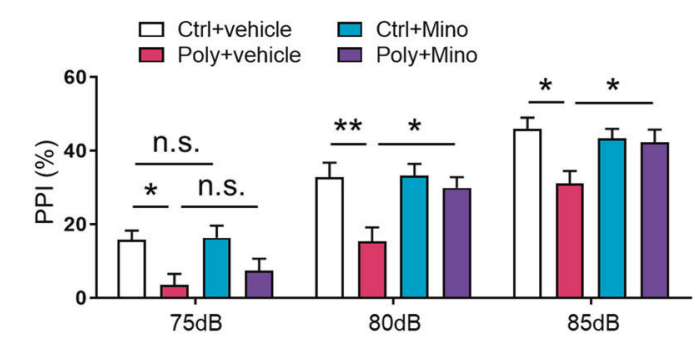

$\mathbf{F}$

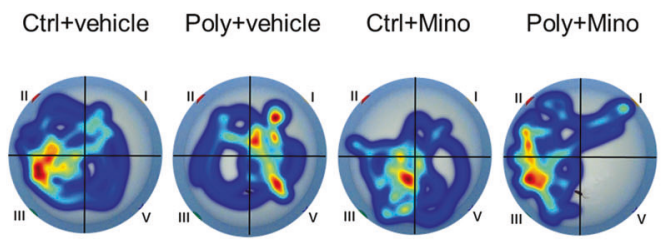

B

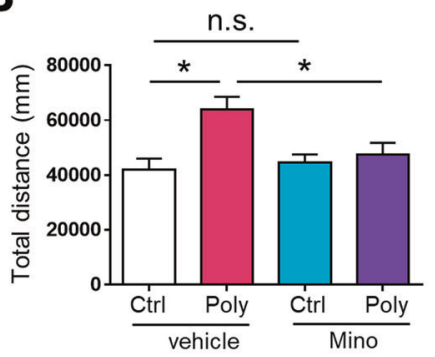

C

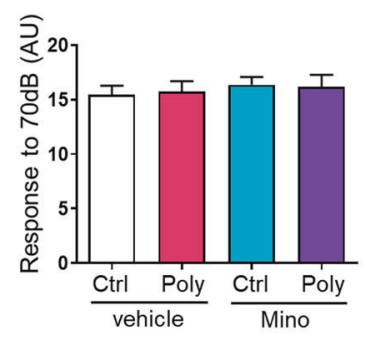

E

$\square$ Nonsocial cylinder - stimulate mice $\square$ Social cylinder - stimulate mice Nonsocial cylinder + stimulate mice $\square$ Social cylinder + stimulate mice

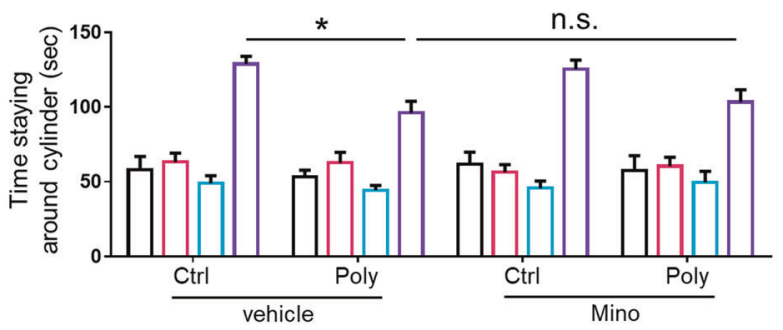

G $\theta$ Ctrl+vehicle $\rightarrow$ Ctrl+Mino $\rightarrow$ Poly+vehicle - Poly+Mino

H
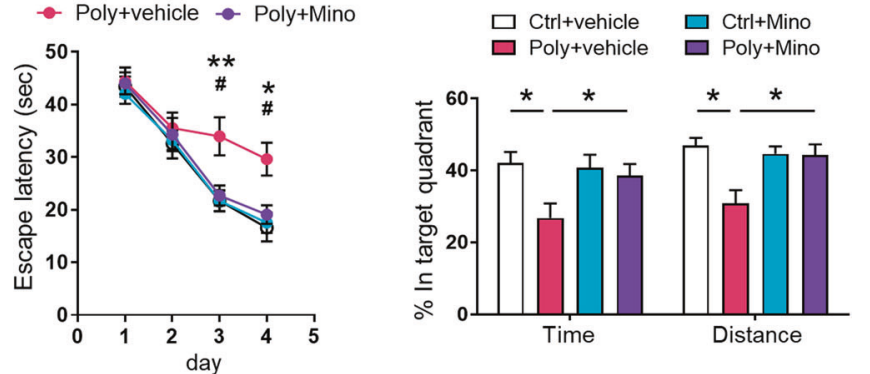

Fig. 1 Minocycline administration ameliorates schizophrenia-like behavior of adult offspring exposed to MIA. a Diagram of poly (I:C) insult, minocycline administration and behavior test for wild-type mice. $\mathbf{b}$ Travel distance in the open field was increased in Poly+vehicle offspring, compared to Ctrl+vehicle offspring, and recovered after minocycline treatment. $n=11$ mice per group; $F_{3,40}=3.008, P<0.05$, oneway ANOVA. c Similar response to $70 \mathrm{~dB}$ background noise between the three groups. $n=11$ per group; $F_{3,40}=0.942, P>0.05$, one-way ANOVA. d PPI was impaired in Poly+vehicle offspring compared with Ctrl+vehicle offspring and ameliorated in Poly + Mino offspring. $n=11$ mcie per group. Group: $F_{3,40}=5.437, P<0.05$; Prepulse intensity: $F_{2,80}=23.82, P<0.001$; Group $\times$ prepulse intensity: $F_{6,80}=1.073, P>0.05$, two-way ANOVA with repeated measures. e Poly+vehicle offspring spent less time around social cylinder with stimulus mouse relative to Ctrl + vehicle offspring, however, the interaction time was failed to improve after minocycline treatment. $n=11$ mice per group; $F_{3,40}=0.374, P>$ 0.05, one-way ANOVA. f Representative moving tracks in the Morris task. $\mathbf{g}$ Increased escape latency for Poly+vehicle group to locate the hidden platform, compared to Ctrl+vehicle offspring, while decreased in Poly+Mino offspring. $n=11$ mice per group. Group: $F_{3}, 40=9.374$, $p<0.05$; Day: $F_{3,120}=17.442, p<0.001$; Group $\times$ day: $F_{9,120}=1.694, p>0.05$, two-way ANOVA with repeated measures. $\mathbf{h}$ In the probe trial, data are presented as percent time and percent distance in the platform quadrant. $n=11$ mice per group; $F_{3}, 40=4.940, P<0.05$, one-way ANOVA. For $\mathbf{g}^{*} p<0.05$, ${ }^{* *} p<0.01$ for Poly+vehicle offspring vs. Ctrl+vehicle offspring; ${ }^{\#} p<0.05$ for Poly+Mino offspring vs. Poly + vehicle offspring. Values are the mean \pm SEM. ${ }^{*} p<0.05,{ }^{* *} p<0.01$, n.s., not significant.

deficits effectively (Fig. S1). To further determine whether the above abnormal behavior reversed by minocycline treatment is schizophrenia-relevant, we administrated clozapine (an antipsychotic medication) to poly (I:C) offspring. We found that hyperactivity and PPI deficits were ameliorated in poly (I:C) offspring (data not shown).

Minocycline treatment ameliorated the deficiency of PV expression and GABAergic transmission onto DGCs of poly $(\mathrm{I}: \mathrm{C})$ offspring

Abnormalities in GABAergic interneurons and inhibitory synaptic transmission in the forebrain, particularly in fast-spiking interneurons that express PV, are associated with schizophrenia-like behavior [48, 49]. To explore the mechanism involved, we first investigated the expression of PV protein in both the hippocampus and $\mathrm{mPFC}$ in offspring mice on PD56. PV protein levels were significantly lower in Poly+vehicle offspring compared with Ctrl +vehicle offspring, which was markedly elevated by minocycline administration in Poly+vehicle offspring in the hippocampus (Fig. 2a). By contrast, poly $(\mathrm{l}: \mathrm{C})$ or minocycline administration did not influence PV levels in the mPFC (Fig. 2b). Consistent with the homogenate data, the numbers of PV + cells were significantly decreased in the DG of Poly+vehicle offspring, and this deficiency was improved by minocycline treatment (Fig. 2c, d). Nevertheless, poly $(I: C)$ or minocycline administration did not influence the number of PV+ cells in the CA1 (Fig. S2A). In addition, we found that the expression of calretinin- or somatostatin-expressing neurons showed no change (data not shown). These results 
A

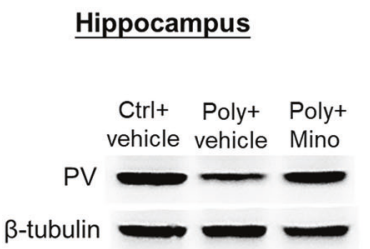

C
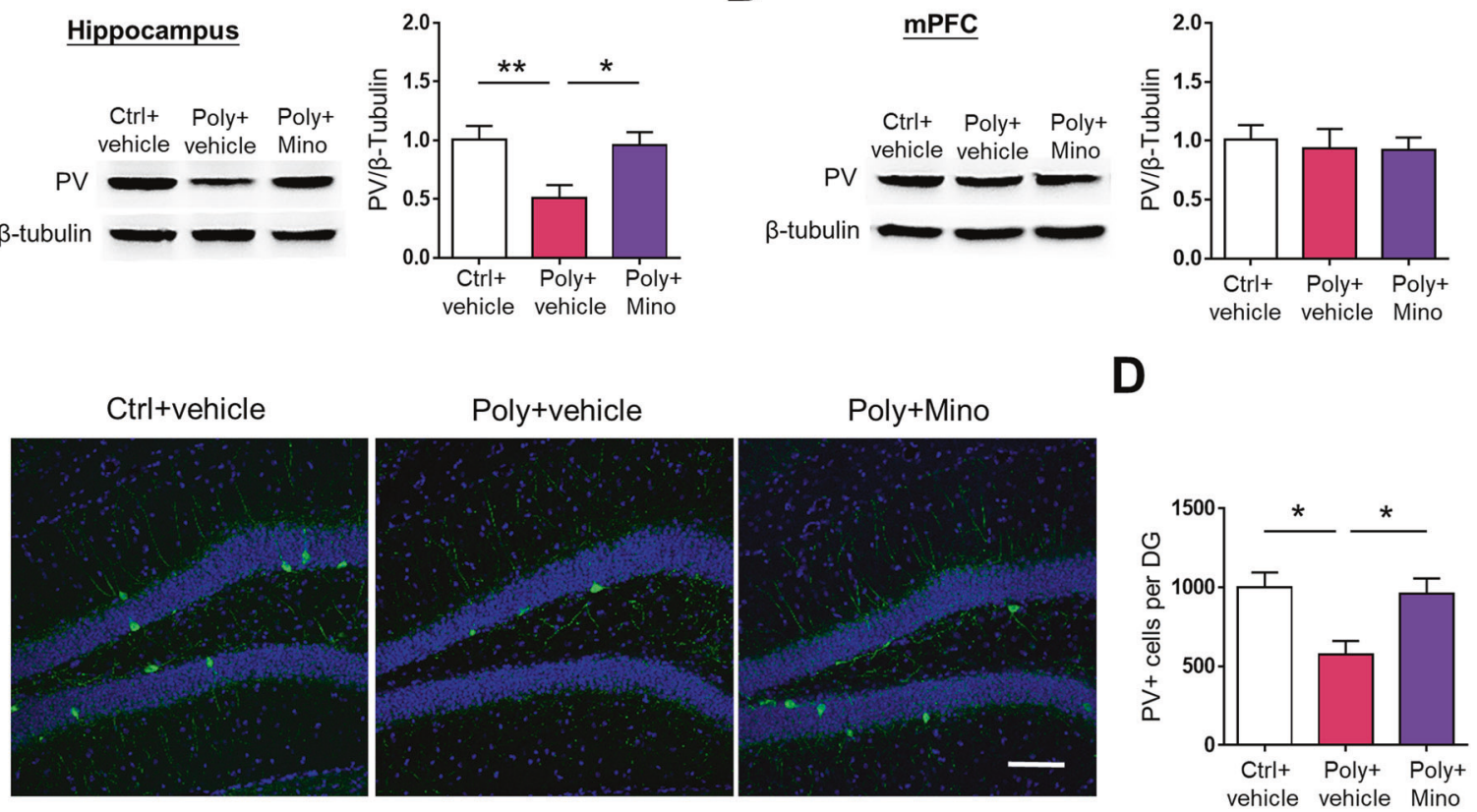

E

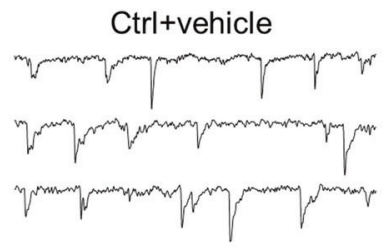

$\mathbf{F}$
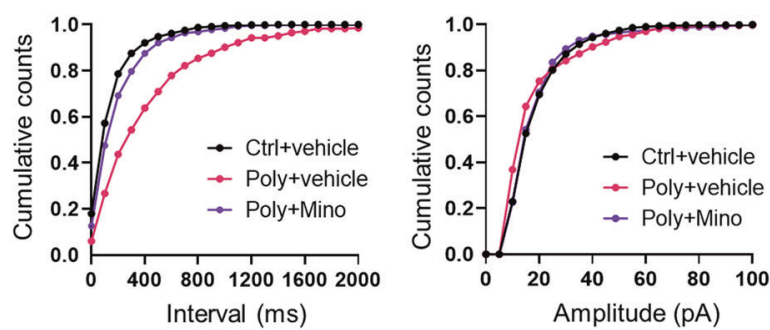

I

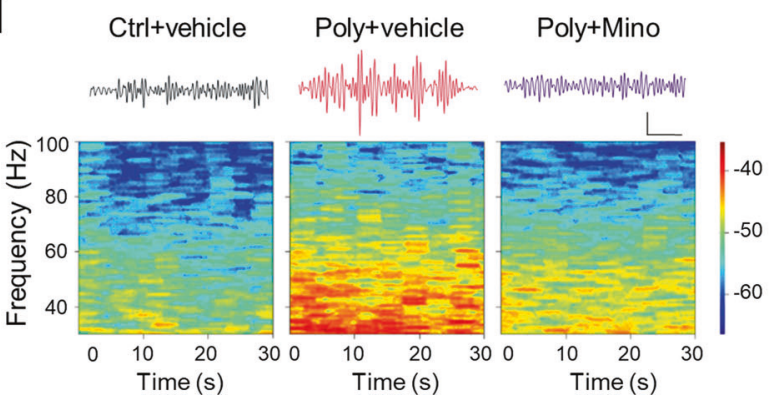

suggest that minocycline reversed the deficiency of PV expression in Poly+vehicle offspring, which may function in behavior deficits.

To better understand the mechanism underlying neuropsychiatric disorders, we next investigated GABAergic transmission by recording mIPSCs in both GCs in DG and CA1 pyramidal cells. As shown in Fig. 2e-h, mIPSC frequency was significantly reduced in

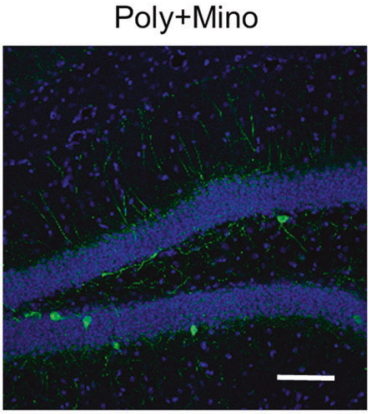

B

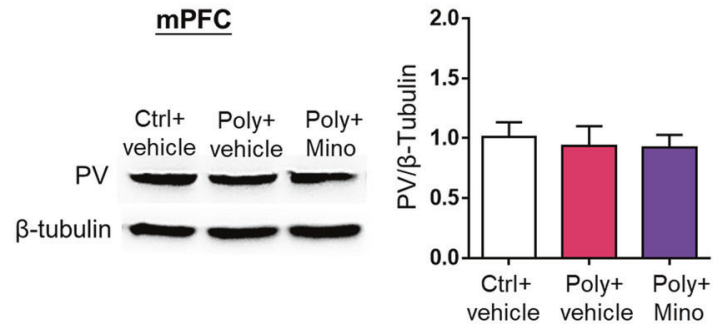

D vehicle vehicle Mino

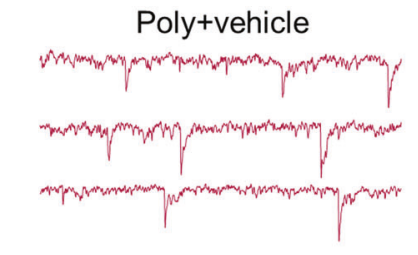

G

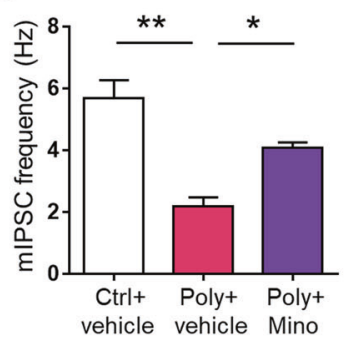

$\mathbf{J}$

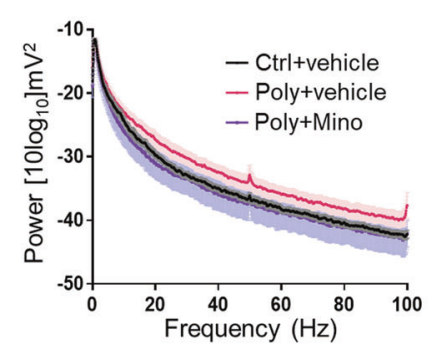

H

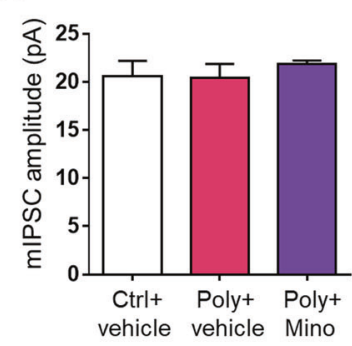

K

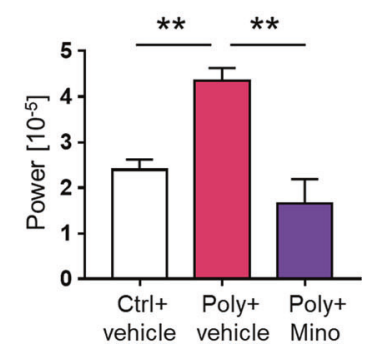

Poly+vehicle offspring compared with Ctrl+vehicle offspring, which suggests many fewer inhibitory synapses or diminished release probability. Nevertheless, the MIA-induced deficits of GABAergic transmission were ameliorated by minocycline administration. By contrast, there was no change in mIPSC amplitude, suggesting GABA receptor density was not compromised. 
Fig. 2 Minocycline treatment attenuates MIA-induced deficits in PV expression and GABAergic transmission onto GCs of the offspring. a Western blot analysis revealed a decreased PV expression levels in the hippocampus of Poly+vehicle offspring relative to the Ctrl+vehicle offspring, and upregulated in Poly+Mino offspring. $n=6$ mice per group; $F_{2,15}=5.759, P<0.05$, one-way ANOVA. b There is no significant difference of PV expression in the mPFC between the three groups. $n=6$ mice per group; $F_{2}, 15=0.166, P>0.05$, one-way ANOVA. c Representative micrographs of PV+ interneurons (green) in the DG. Scale bars: $100 \mu \mathrm{m}$. $\mathbf{d}$ The total number of PV+ cells were reduced in Poly+vehicle offspring, and the defect was improved in Poly+Mino offspring. $n=6$ mice per group; $F_{2,15}=4.064, P<0.05$, one-way ANOVA. e Representative mIPSC traces in hippocampal slices from Ctrl+vehicle, Poly+vehicle, and Poly+Mino offspring. Scale bars, $200 \mathrm{~ms}$ and 20 pA. f Cumulative plots of mIPSC frequencies. g Attenuated mIPSC frequency of granule cells in Poly+vehicle offspring compared to Ctrl+vehicle offspring, whereas ameliorative in Poly+Mino offspring. $n=15$ cells from 5 mice per group; $F_{2,42}=8.637, P<0.05$, one-way ANOVA. $h$ mIPSC amplitudes were similar within Ctrl+vehicle, Poly+vehicle and Poly+Mino offspring. $n=15$ cells from 5 mice per group; $F_{2,42}=0.948, P>0.05$, one-way ANOVA. i Minoycline rescued the hypersynchronic activity in Poly+vehicle offspring. Top, example gamma-band traces from hippocampus. Scale bar, $200 \mathrm{~ms}$ and $0.5 \mathrm{mV}$. Bottom, spectrograms from hippocampus. j Power spectrum of DG LFP from 0.5-100 Hz (shaded areas, SEM). k Average relative LFP power in gamma frequency bands $(30-80 \mathrm{~Hz})$ of different groups. $n=6$ mice per group; $F_{2,15}=20.58, P<$ 0.01 , one-way ANOVA. ${ }^{*} p<0.05,{ }^{* *} p<0.01$. Values are the mean \pm SEM.

Moreover, neither the frequency nor amplitude of the mIPSCs onto pyramidal cells significantly differed between these groups (Fig. S2B-D).

To further confirm whether minocycline treatment regulates the $\mathrm{PV}+$ interneurons-induced GABAergic transmission in Poly+vehicle offspring, we detected the gamma oscillations, whose production has been closely related to PV+ cell activity $[50,51]$. As shown in Fig. 2i-k, Poly+vehicle offspring had hypersychronic activity and enhanced gamma oscillations in the DG, compared to Ctrl+vehicle offspring. Minocycline treatment effectively reversed the gamma oscillations. In conclusion, the above results suggest that minocycline treatment regulated PV expression, GABAergic transmission, and increased gamma oscillations in the DG of Poly+vehicle offspring.

Minocycline treatment rescues neuroinflammation as well as microglia Arg1 expression in the hippocampus of poly $(\mathrm{I}: \mathrm{C})$ offspring

Accumulating evidence indicates that neuroinflammation in the CNS is directly related to behavioral deficits and synaptic dysfunction in pathologic processes of psychiatric disorders, including upregulation of inflammatory mediators and microglia activation [52, 53]. To investigate the neuroinflammation induced by MIA, the levels of pro-inflammatory cytokines such as IL-1 $\beta$, TNF- $\alpha$, and IL- 6 in the hippocampus were evaluated. As shown in Fig. $3 a-d$, the expression of IL-1 $\beta$, TNF- $\alpha$, and IL- 6 was significantly increased in the hippocampus of Poly+vehicle offspring, while these pro-inflammatory cytokines were attenuated by minocycline treatment. Next, we examined the effect of minocycline on microglia activation by immunostaining. As shown in Fig. $3 \mathrm{e}-\mathrm{g}$, the total numbers of $\mathrm{Iba} 1+$ cells were markedly increased in the hippocampus of Poly+vehicle offspring and were restored by minocycline treatment. Furthermore, an activated morphology, with longer branched processes of $\mathrm{lba} 1+$ microglia was found in Poly+vehicle offspring exhibiting hyper-ramification activation, whereas it was blocked by Minocycline treatment. In contrast, the number of primary processes and soma size of Iba1+ microglia were comparable between groups (Figs. S3A and 3B). In addition, we investigated the number of astrocytes, another type of resident immune cell in the CNS. There was no significant difference in the total numbers of GFAP + cells in the hippocampus of the three groups (Fig. S3C). Taken together, these results suggest that neuroinflammation accompanied by microglia activation induced by MIA can be effectively alleviated by minocycline treatment.

To figure out the role of Arg1 in MIA-induced microglia activation improved by minocycline administration, we first tested protein levels of both Arg1 and Ym1 (Fig. 3h). Arg1 protein expression was much lower in Poly+vehicle offspring than Ctrl+vehicle offspring, whereas minocycline treatment significantly reversed the levels of Arg1 (Fig. 3i). By contrast, Ym1 levels were not obviously different between these groups (Fig. 3j). In agreement with the changes in protein levels, Arg1 immunoreactivity was markedly lower in Poly+vehicle offspring relative to $\mathrm{Ctrl}+$ vehicle offspring and was obviously promoted after minocycline treatment (Fig. 3k, I, Fig. S3D). Together, these results suggest that Arg1 is related to minocycline administration for microglia activation induced by MIA.

Specific ablation of Arg1 in microglia prevents minocycline ameliorated schizophrenia-like behavior of adult poly $(\mathrm{I}: \mathrm{C})$ offspring

Considering the critical role played by Arg 1 in microglia activation, we determined whether microglia Arg1 contributes to the psychiatric deficits in poly $(I: C)$ offspring with or without minocycline. To this end, floxed Arg1 mice were crossed with CX3CR1-creER mice to generate mice lacking Arg1-specific microglia until TAM injection (CX3CR1-Arg $1^{\mathrm{f} / \mathrm{f}}$ ) (Fig. 4a). As shown in Fig. 4b, Arg1 expression in the hippocampus was significantly reduced in CX3CR1-Arg $1^{\mathrm{f} / \mathrm{f}}$ mice, compared to $A r g 1^{\mathrm{f} / \mathrm{f}}$ littermate mice. CX3CR1-Arg1 ${ }^{\mathrm{f} / \mathrm{f}}$ pregnant mice were subjected to poly $(\mathrm{I}: \mathrm{C})$ on GD12 as before (Fig. 4c). Similarly, CX3CR1-Arg1/f offspring from the poly $(I: C)$ injected mothers exhibited schizophrenia-like deficits in a series of behavior tests as before. However, minocycline failed to improve behavior deficits in the CX3CR1$\operatorname{Arg}_{1} / \mathrm{f}$ offspring induced by MIA (Fig. $4 d-h$ ). There was no difference in above-mentioned behavior tests under both control and MIA states between offspring from CX3CR1-Arg $1^{\mathrm{f} / \mathrm{f}}$ and $\mathrm{Arg} 1^{\mathrm{f} / \mathrm{f}}$ mice. These results suggest that ablation of Arg1 in microglia can dampen the effect of minocycline action on schizophrenia-like behavior caused by MIA.

Microglia Arg1 overexpression in the DG ameliorates schizophrenia-like behavior of adult poly (I:C) offspring To further identify the critical role of DG microglia Arg1 on the effects of minocycline treatment of behavior deficits induced by MIA, Arg1 was overexpressed by stereotactic injection with AAVDIO-Arg1-GFP in bilateral DG of CX3CR1-creER offspring from poly $(I: C)$ injected mothers (Fig. 5a, b). As shown in Figs. $5 c$ and S4A, the majority of GFP + cells were co-labeled with lba1+ cells $(74.61 \pm$ $4.72 \%$ of $\mathrm{lba} 1+$ cells) in AAV-DIO-Arg1-GFP mice. To further identify whether Arg1 is upregulated in GSs of DG following injection, we isolated microglial cells by fluorescence-activated cell sorter following AAV stereotactic injection in CX3CR1-creER mice, then detected the mRNA level of Arg1 by qRT-PCR. As shown in Fig. S4B and $C$, the mRNA level of Arg1 was upregulated in Poly+AAV-DIO-Arg1-GFP group compared with Poly+AAV-emptyGFP and Ctrl groups. We next performed the same behavior tests used in Fig. 1 and observed that both Poly and Poly+AAV-emptyGFP offspring displayed hyperactivity, and impaired PPI and spatial memory. More importantly, all above-mentioned behavior deficits were reversed in Poly+AAV-DIO-Arg1-GFP offspring (Fig. $5 \mathrm{~d}-\mathrm{h}$ ). In short, our results indicate that microglia Arg1 upregulation in DG rescued the impaired schizophrenia-relevant behavior in MIA offspring. 
A

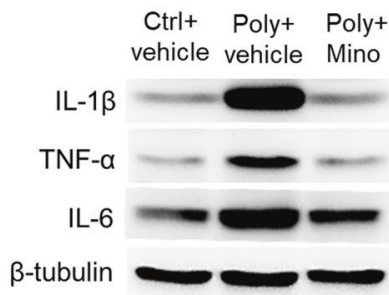

E

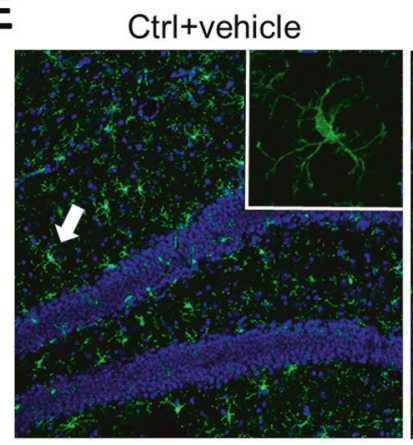

G

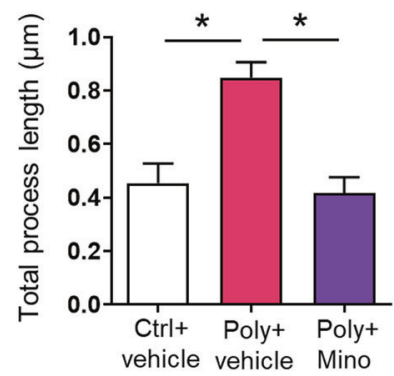

K

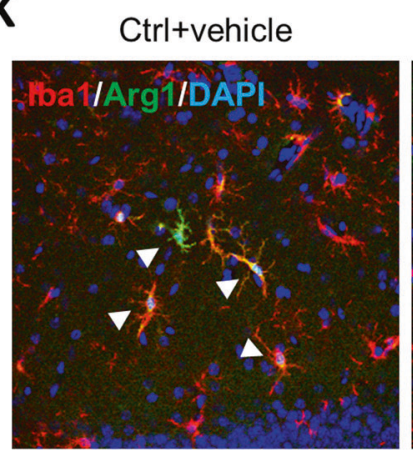

B

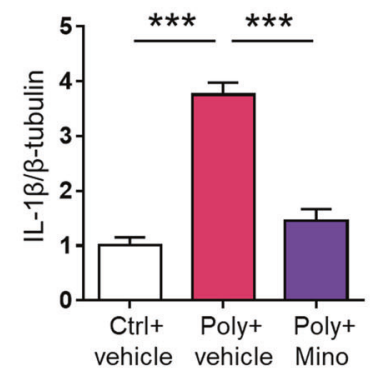

Poly+vehicle

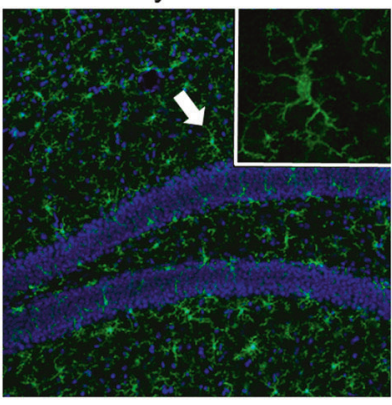

H

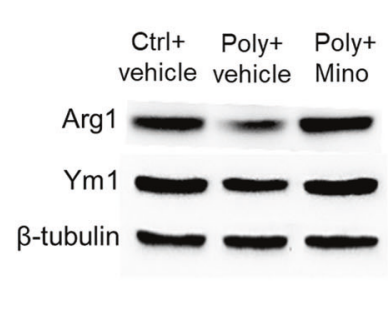

C

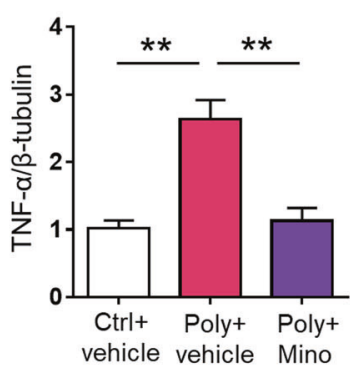

Poly+Mino

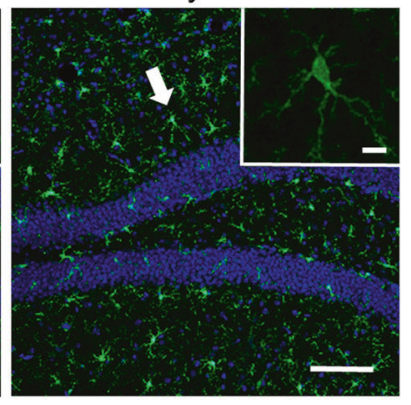

I

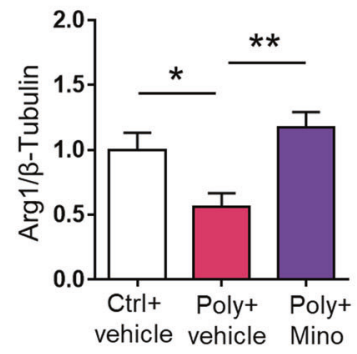

Poly+vehicle

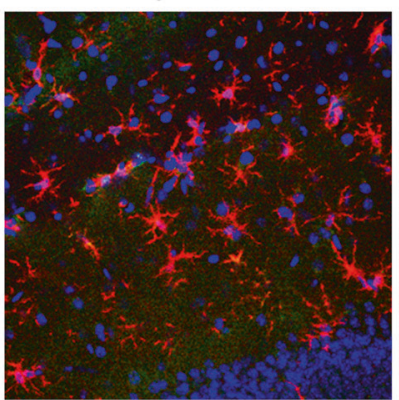

Poly+Mino

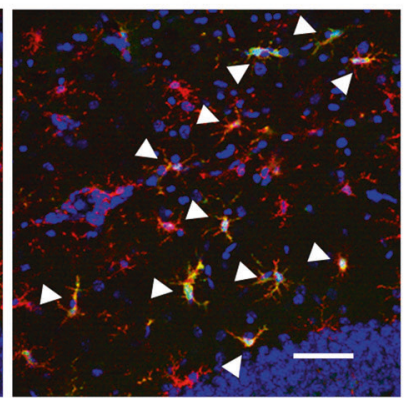

D

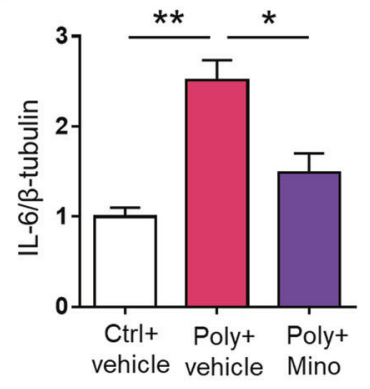

F

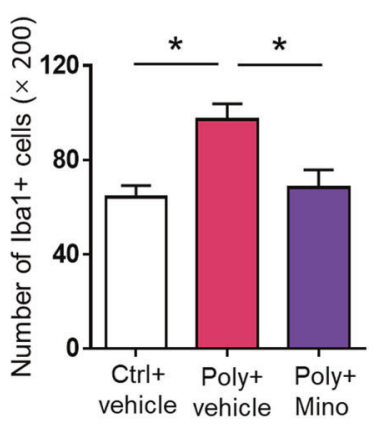

J

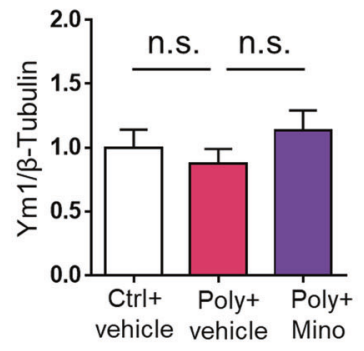

L

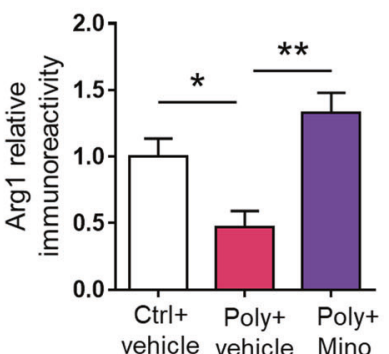

Fig. 3 Minocycline treatment reduces neuroinflammation and upregulates the hippocampus Arg1 expression in activated microglia of offspring mice exposed to MIA. a Representative western blot films for IL-1 $\beta$, TNF- $\alpha$, and IL- 6 protein expression in the hippocampus of the offspring. The corresponding quantification of $\mathbf{b} \mathrm{IL}-1 \beta, \mathbf{c}$ TNF- $\alpha$, and $\mathbf{d} \mathrm{IL}-6$ in the offspring mice. $n=6$ per group; IL-1 1 : $\mathrm{F}_{2,15}=18.93, P<0.001$; TNF- $\alpha: F_{2,15}=15.24, P<0.01 ; \mathrm{IL}-6: F_{2,15}=2.95, P<0.05$, one-way ANOVA. e Representative micrographs of Iba $1+$ cells (green) in the DG. Scale bars: $100 \mu \mathrm{m}$. White arrows indicate the cell represented in the inset. Scale bars: $10 \mu \mathrm{m}$. $\mathbf{f}$ Number of Iba-1+ cells $(\times 20)$ in the DG of the offspring. $n=6$ mice per group; $F_{2,15}=9.34, P<0.05$, one-way ANOVA. $g$ Quantitative analysis for mean ramification index of microglia. $n=6$ mice per group; $F_{2,15}=6.546, P<0.05$, one-way ANOVA. $h$ Representative western blot films for Arg1 and Ym 1 protein expression in the hippocampus of the offspring. The corresponding quantification of $\mathbf{i}$ Arg1 and $\mathbf{j}$ Ym1 expression levels in the offspring. $n=6$ mice per group; Arg1: $F_{2,15}=5.475, P<0.05 ;$ Ym1: $F_{2,15}=1.058, P>0.05$, one-way ANOVA. k Representative fluorescent images for Arg1 immunoreactivity in the DG of offspring mice. Scale bars: $20 \mu \mathrm{m}$. I Quantification of Arg1 immunoreactivity (in arbitrary units) in the DG. $n=6$ mice per group; $F_{2,15}=4.197, P<0.05$, one-way ANOVA. ${ }^{*} p<0.05,{ }^{* *} p<0.01,{ }^{* *} p<0.001$, n.s., not significant. Values are the mean \pm SEM. 
A

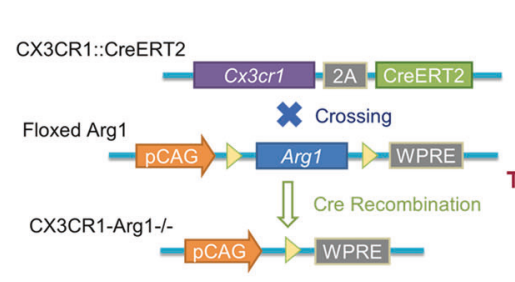

C

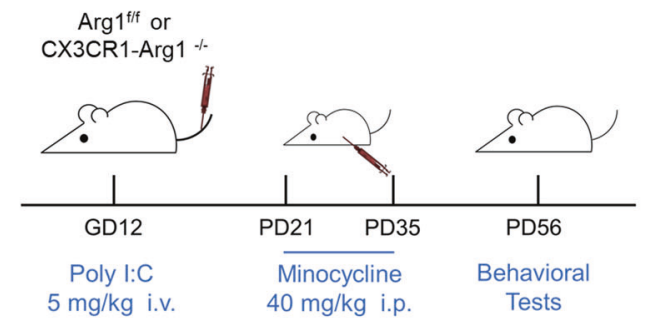

B

CX3CR1-Arg1 1 -

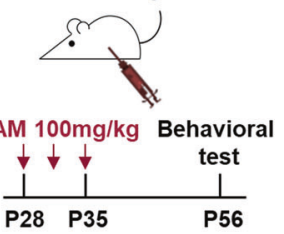

E

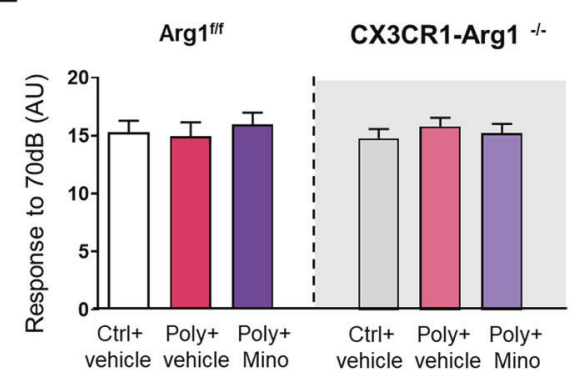

G

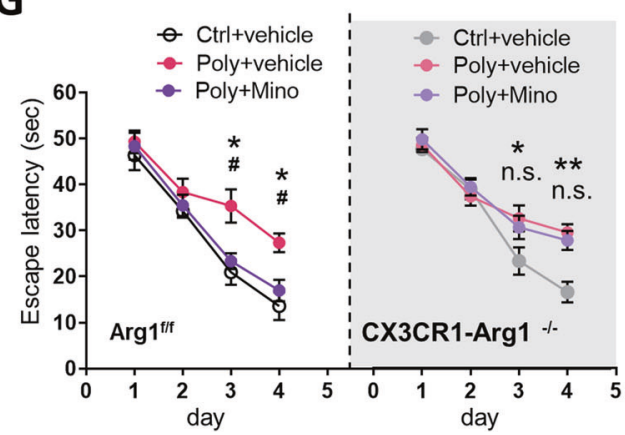

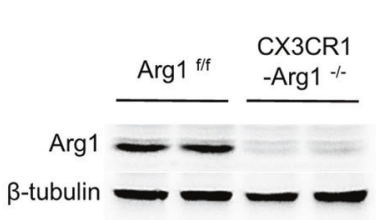

D

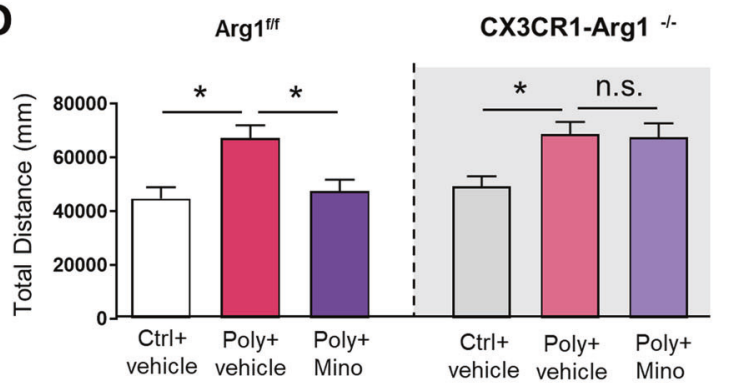

$\mathbf{F}$

$\operatorname{Arg}^{\mathrm{fif}}$

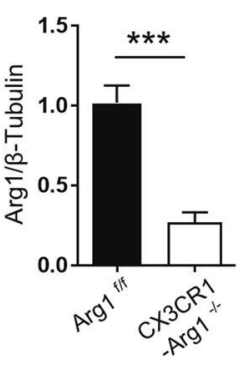

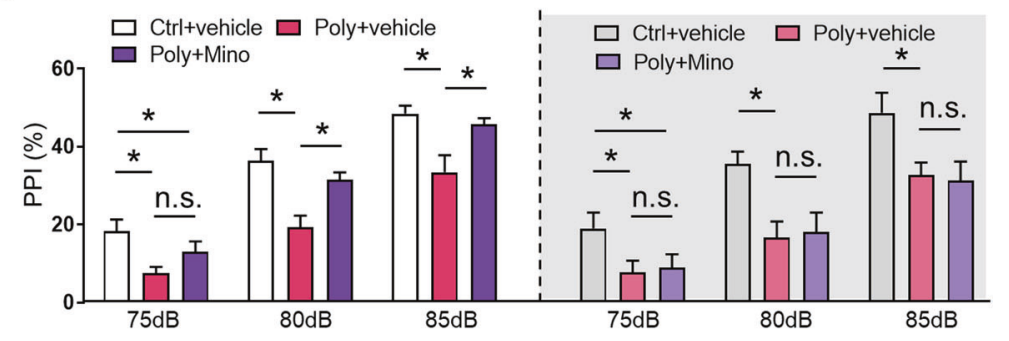

H

$\operatorname{Arg} 1^{1 / f}$

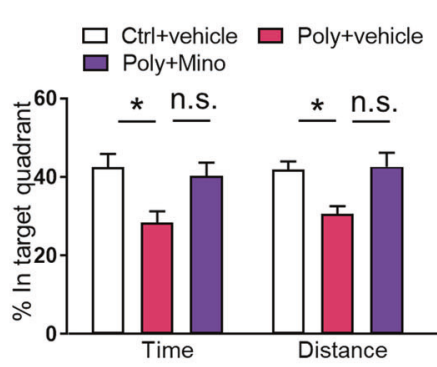

CX3CR1-Arg1 --

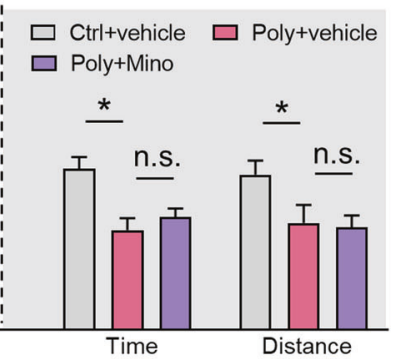

Fig. 4 Ablation of Arg1 in Microglia prevents minocycline ameliorating behavior deficits of adult offspring exposed to MIA. a Mice expressing the two transgenes were crossed and injected with tamoxifen. $\mathbf{b}$ Arg1 protein level was obviously reduced in the hippocampus of CX3CR1-Arg $1^{-/-}$offspring compared with the Arg $1^{\mathrm{f} / \mathrm{f}}$ littermates. $n=5$ per group; $F_{1,8}=18.75, P<0.001$, one-way ANOVA. c Diagram of poly $(\mathrm{I}: C)$ insult, minocycline treatment and behavior test for CX3CR1-Arg $1^{-/}$mice and Arg $1^{\mathrm{f} / \mathrm{f}}$ littermates. $\mathbf{d}$ Total distance of adult offspring mice in open field test. $n=10$ per group; Group: $\mathrm{F}_{2,27}=4.852, P<0.01$, two-way ANOVA. e Similar response to $70 \mathrm{~dB}$ background noise in the three group. $n=10$ mice per group; Group: $F_{2,27}=0.944, p>0.05$, two-way ANOVA. $f$ PPI was impaired in Poly+vehicle offspring of CX3CR1-Arg ${ }^{-1-}$ mice compared to Ctrl+vehicle offspring and failed to ameliorate in Poly+Mino offspring. $n=10$ mice per group; Group: $F_{2,27}=10.274, P<$ 0.01 , prepulse intensity: $F_{2,54}=26.308, P<0.001$; Group $\times$ prepulse intensity: $F_{4,54}=1.773, P>0.05$, three-way ANOVA with repeated measures. $g$ Increased latency for Poly+vehicle offspring of CX3CR1-Arg $1^{-1-}$ mice to reach the hidden platform compared with Ctrl+vehicle offspring and failed to restore in Poly+Mino mice. $n=10$ mice per group; Group: $F_{2,27}=7.670, p<0.01 ;$ day: $F_{3,81}=19.035, p<0.001$; Group $\times$ day: $F_{6,81}=1.553, p>0.05$; three-way ANOVA with repeated measures. $\mathbf{h}$ In the probe trial, data are presented as percent time and percent distance in the target quadrant. $n=10$ mice per group; Group: $F_{2,27}=6.298, p<0.01$, two-way ANOVA. For all figures, there is no "Genotype" or "Genotype $\times$ Group" interactions were detected (all $p$ 's $>0.05$ ). For Fig. $g^{*} p<0.05,{ }^{* *} p<0.01$, ${ }^{* * *} p<0.001$ for Poly + vehicle offspring vs. Ctrl+vehicle offspring, ${ }^{\#} p<0.05$ for Poly+Mino offspring vs. Poly+vehicle offspring. Values are the mean \pm SEM. ${ }^{*} p<0.05,{ }^{* *} p<$ $0.01,{ }^{* *} p<0.001$, n.s., not significant. 
A PAAV

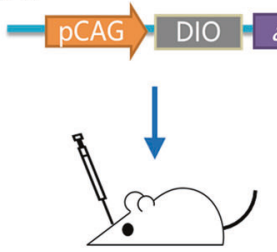

CX3CR1-creER

C

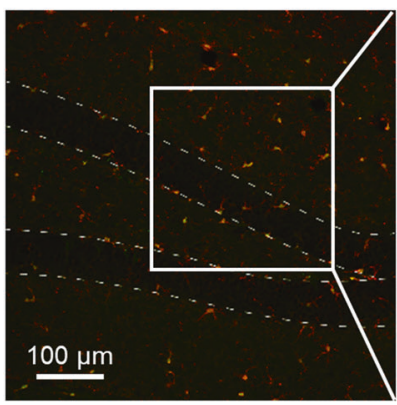

D

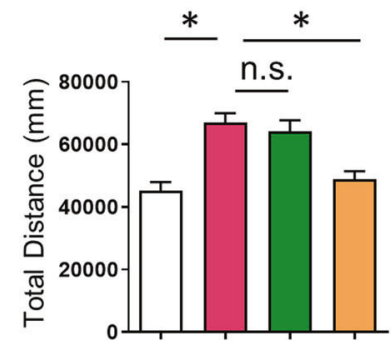

B

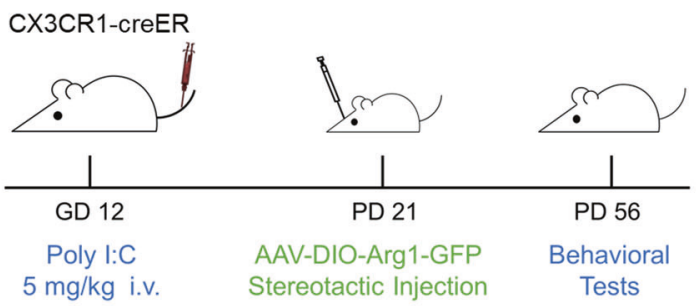

G

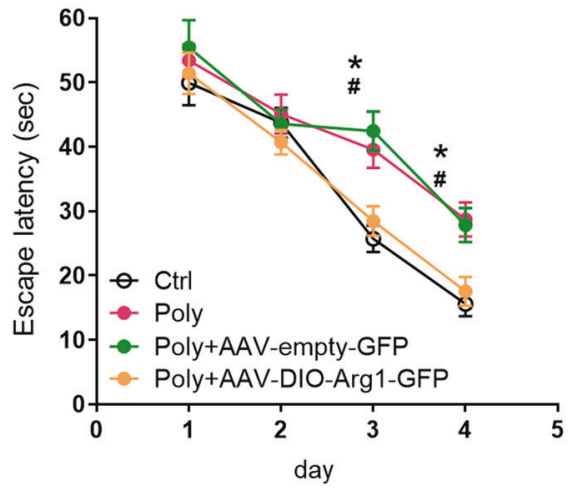

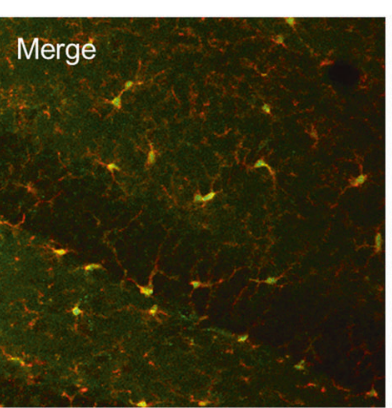

E

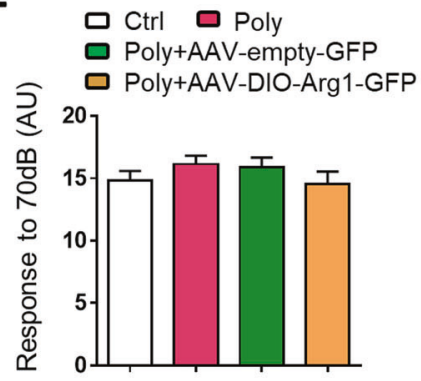

H

\section{H}
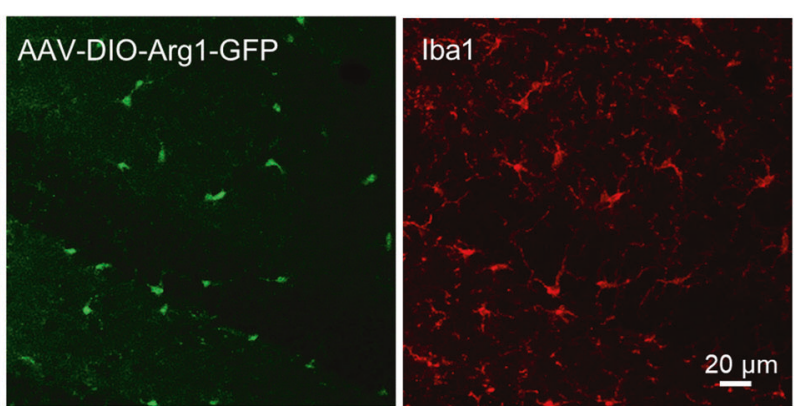

F

$\square$ Ctrl $\square$ Poly+AAV-empty-GFP $\square$ Poly $\square$ Poly+AAV-DIO-Arg1-GFP

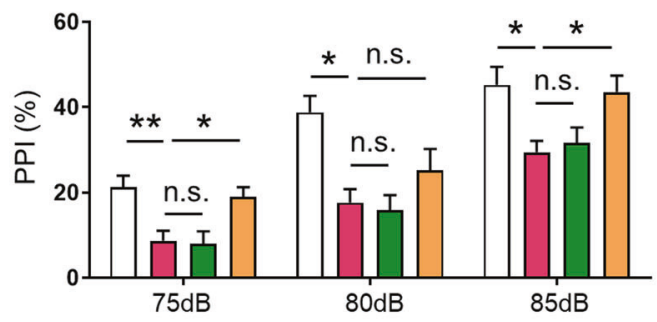

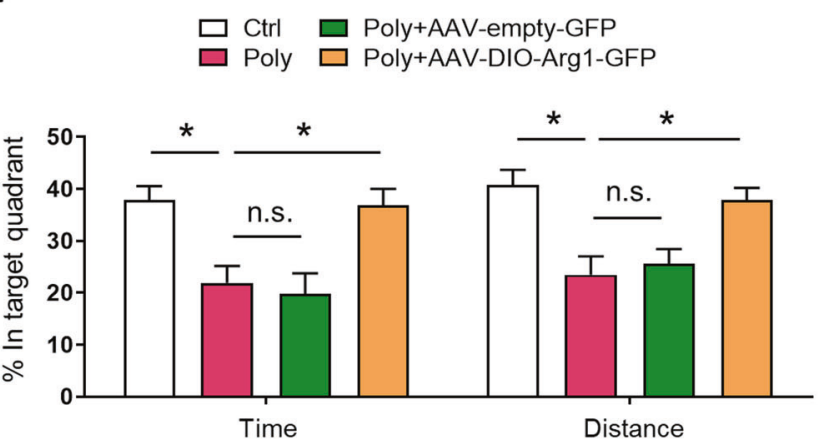

Fig. 5 Microglia-specific Arg1 overexpression in hippocampus DG ameliorates schizophrenia-like behavior of adult offspring exposed to MIA. a Schematic diagram showing the experimental strategy for Arg1 overexpression. AAV-DIO-Arg1-GFP was stereotactic injected into hippocampus DG of CX3CR1-creER mice. b Timeline of experiment. Mice were analyzed 5 weeks after AAV injection. c Representative micrographs of Arg1 overexpression characteristically in hippocampal microglia. $\mathbf{d}$ Total distance of the adult offspring in open field test. $n=$ 10 per group; $F_{3,36}=5.471, P<0.05$, one-way ANOVA. e Similar response to $70 \mathrm{~dB}$ background noise in the three group. $\mathbf{f P P I}$ was impaired in Poly offspring compared with Ctrl offspring and ameliorated in Poly+AAV-DIO-Arg1-GFP offspring. $n=10$ mice per group. Group: $F_{3,36}=$ 8.963, $P<0.05$; Pepulse intensity: $F_{2,72}=18.61, P<0.001$; Group $\times$ Prepulse intensity: $F_{6,72}=0.554, P>0.05$, two-way ANOVA with repeated measures. $g$ Increased latency for Poly offspring to locate the hidden platform, compared to Ctrl offspring, and decreased in Poly + AAV-DIOArg1-GFP offspring. $n=10$ mice per group. Group: $F_{3,36}=4.873, p<0.05$; Day: $F_{3,108}=13.719, p<0.01 ; G$ roup $\times$ day: $F_{9}, 108=2.824, p>0.05$, two-way ANOVA with repeated measures. $\mathbf{h}$ In the probe trial, data are presented as percent time and percent distance in the target quadrant. $n=10$ mice per group, $F_{3,36}=3.568, P<0.05$, one-way ANOVA. For Fig. ${ }^{*} p<0.05,{ }^{* *} p<0.01$ for Poly offspring vs. Ctrl offspring; ${ }^{\#} p<0.05$ for Poly+AAV-DIO-Arg1-GFP offspring vs. Poly offspring. Values are the mean \pm SEM. ${ }^{*} p<0.05,{ }^{* *} p<0.01$, n.s., not significant. 
Microglia Arg1 overexpression in DG ameliorates GABAergic functional deficits and microglia activation of adult poly (I:C) offspring

To better understand the effect of upregulation of microglia Arg1 in the pathology of psychiatric disorders, we next investigated PV + cell number and GABAergic transmission onto GCs in DG. Under microglia Arg1 upregulation, the reduced PV+ cells induced by MIA were significantly rescued (Poly+AAV-DIO-Arg1-GFP offspring), compared to Poly+AAV-empty-GFP offspring (Fig. S5A). We next investigated GABAergic transmission by recording mIPSCs in GCs. Similar to immunostaining evidence, the decreased mIPSC frequency in poly $(I: C)$ offspring can be notably attenuated by injection of AAV-DIO-Arg1-GFP, but not by AAV-empty-GFP (Fig. S5B-E). Furthermore, the total numbers of Iba-1+ cells were markedly increased in the hippocampus of poly (I:C) offspring and were restored by Arg1 overexpression (Fig. S6). Altogether, these results led us to conclude that microglia Arg1 upregulation in DG rescue the deficiency of PV+ cells and GABAergic synaptic transmission, which relate to microglia activation.

\section{DISCUSSION}

In this study, we provide further insights into a pathophysiological mechanism for MIA-induced deficits and a potential target for intervention. First, we found deficits in PV expression in DG and GABAergic synaptic transmission onto GCs, along with schizophrenia-like behavior of the poly $(I: C)$ offspring mice. Pharmacologically, intervention of microglia activation attenuates neuropathological damages exposed by MIA. Genetically, we provide evidence that microglia Arg1 in DG are necessary for prenatal poly $(\mathrm{I}: \mathrm{C})$ injection induced neuropathology disorder.

Our results showed that the number of $\mathrm{PV}+$ interneurons in DG was reduced in poly ( $\mathrm{I}: \mathrm{C})$ offspring (Fig. 2a, c), along with decreased frequency of mIPSC of GCs (Fig. 2e-h). Consistent with our results, a recent study also reported that MIA decreased the number of $\mathrm{PV}+$ interneurons in the primary somatosensory cortex along with a reduction in frequency of mIPSCs pyramidal neurons in MIA offspring [54]. Furthermore, another study found lower frequency of mIPSC and decreased light-evoked PV GABAgergic transmission onto pyramidal cells in MPFC of adult MIA offspring [55]. However, our western Blot results showed that the expression of PV in MPFC had not been altered in MIA offspring (Fig. 2b). We speculate that different effects in MPFC may be due to variations in the intervention time point (GD12 vs. GD9) and/or administration dosage of poly (l:C) $(5 \mathrm{mg} / \mathrm{kg}$ vs. $1 \mathrm{mg} / \mathrm{kg})$. Besides, we found that two weeks minocycline treatment at the third postnatal week from PD21 prevented the loss of DG PV + neurons in MIA mice (Fig. 2c, d). In schizophrenia patients, increased levels of immature neuronal progenitors and a deficiency in mature neurons have been found in the DG, indicating impaired hippocampal neurogenesis [56]. Consistent with our results, previous studies showed that minocycline rescues the decrease in neurogenesis and increase in microglia activation $[57,58]$. Thus, we speculate that the potential mechanism of the $\mathrm{PV}+$ interneurons loss may be associated with hippocampal neurogenesis, which awaits further verification. Together, our results support the idea that neuroinflammation may have important effects on the GABAergic interneuron development and function $[59,60]$.

In this study, our results showed an increased gamma oscillation, accompanied with a profound loss of $\mathrm{PV}+$ neurons in MIA offspring (Fig. 2). It is worth noting that the mechanism of enhanced gamma oscillation in DG may be influenced by multiple factors. On one hand, reduced performant-path (PP) inputs on the dendrites of GCs cells in DG play an important role in the generation of gamma oscillation [61, 62]. We suspected that reduced local inhibition in poly $(I: C)$ offspring will enhance the glutamatergic neurotransmission from PP synapses onto mature GCs, which may result in increased gamma oscillation. On the other hand, elimination of young adult-generated granule cells increases overall DG excitability [63] and gamma network activity [64]. Meanwhile, local PV + interneurons in DG serve as a unique local circuit component to regulate hippocampal neurogenesis $[65,66]$. Thus, reduced PV+ interneurons in DG result in the loss of adult-generated neurons in poly $(I: C)$ offspring, which further causes an increase in gamma oscillation.

Human post-mortem analysis showed that the pathogenesis of schizophrenia is associated with an increased number of activated microglia [12]. In this study, we observed the robust enhancement in number of microglia cells alone with a hyper-ramification (Fig. 3g), indicating an increase in microglia activation. Morphologically, the increased microglia ramifications as an active state have been shown in various chronic inflammation rodent models for impaired mood and cognition [67, 68]. However, amoeboid microglia are usually induced by acute inflammation such as ischemic brain injury [69, 70]. Meanwhile, previous studies reported that MIA could result in offspring chronic inflammation [5], which could be the reason why microglia ramification was the main phenotype observed in our study.

Previous studies identified that the transformation of microglia towards protective phenotypes imparts neuroprotective effects in psychiatric disorders by immunosuppressive drugs or molecules, however, the key underlying molecular mechanism is unknown $[71,72]$. In this study, we first revealed the critical role of Arg1 in regulating protective phenotypes of microglia in a MIA model. Supporting our results, pharmacological inhibition of Arg1 blocked the neuroprotective effect of macelignan cells on inflammatory degeneration of midbrain dopaminergic neurons [73]. In addition, global and myeloid-specific knockout of Arg1 worsened neuronal loss along with increased inflammatory and neurotoxic markers in ischemia reperfusion models [74]. However, CX3CR1 is also expressed in tissue-resident macrophages in peripheral cell types [75], and whether CXCR1 outside the CNS takes part in the brain disorders is still controversial [76, 77]. Considering that poly $(I: C)$ is a systemic manipulation, the current study cannot exclude that Arg1 expressed in other cell types is implicated in the effects of minocycline in MIA offspring.

More importantly, we have shown that Arg1 overexpression in DG leads to the correction of behaviors induced by MIA. Hippocampal DG serves as a primary gate, filtering and processing sensory inputs from neocortical sensory and associational projections [78]. The activity of GCs in DG is required in contextual fear memories, locomotor activity and stress response [79]. Meanwhile, immature GCs in DG have been reported to play important roles in long-term spatial memory in MWM task [80]. Another study has revealed that the survival of GABAergic inhibitory neurons in the DG may be involved in impaired sensorimotor gating such as PPI in an adolescent perturbations model [81]. Together, these behaviors are supported by the function of pattern separation in DG, which is based on the local circuit of reciprocal connectivity among interneurons, GCs, and adult-born neurons [82, 83]. We propose that overexpression of Arg1 in DG microglia may exert neuroprotective effects on the PV+ interneuron development by facilitating microglia into alternative phenotypes. Furthermore, the restoration of these $\mathrm{PV}+$ interneurons greatly enhances the feedforward inhibition onto the GCs by monosynaptic excitation from PP afferents (PP-IN-GC) and promotes feedback inhibition onto the GCs (GC-IN-GC). The above circuits of local inhibition were reported to maintain the sparse activity pattern of the granule cells in the DG [84], which maybe lead to the correction of these behaviors. In summary, our findings could shed light on the pathogenic mechanisms of schizophrenia and provide a new target for more effective therapies in neuropathology disorders (Fig. S7).

While the present study has provided evidence that modulating microglia activation can affect maternal immune activation induced schizophrenia-relevant phenotypes, it has limitations that 
must be acknowledged. First, mice in Ctrl+Mino group were not included in neural analysis. Our results demonstrated that minocycline treatment alone in control mice did not cause abnormal behaviors (Fig. 1), and other previous studies also showed no statistical difference between Ctrl+Mino and Ctrl + vehicle in schizophrenia-relevant behaviors and neural analysis $[24,57,85,86]$. However, the current results do not fully determine whether minocycline influences neural functions under physiological conditions. Second, except behavior tests, only male mice were included in the analysis of neural mechanisms. Although the female offspring exposed to MIA presented the similar behavior deficits as male littermates, which can be prevented by minocycline treatment (Figs. 1 and S1), given that females and male animals may have a similar phenotype mediated through separate mechanisms [87], neural analysis in female offspring needs to be further investigated.

\section{FUNDING AND DISCLOSURE}

This work was supported by National Key R\&D Program of China (Grant 2019YFC1712105 to Y Chen); National Natural Science Fund of China (Grants 31600843 to $Y$ Xia and 81973948 to $Y$ Chen); Guangdong Province Universities and Colleges Pearl River Scholar Funded Scheme (2016, Grant to Y Chen); Science and Technology Program of Guangdong (Grant 2018B030334001 to Y Chen); Research Grant of Guangdong Province Key Laboratory of Psychiatric Disorders (N201801 to Y Chen) and Innovation Team Program of Guangdong Provincial Department of education (2018KCXTD006 to Y Chen and 2017KCXTD006 to N Xu); Science and Technology Program of Guangzhou, China (Grant No: 202002030190 to $\mathrm{L}$ Yao). The authors declare no conflict of interest.

\section{AUTHOR CONTRIBUTIONS}

Y. Xia, Y. Chen, L. Yao, N. Xu, and R.W. Caldwell designed the experiments and wrote the paper, Y. Xia, D. Yin, Z. Zhang, W. Lin, J. Yan, C. Zhu, S. He, and Y. Su performed the experiments and analyzed data.

\section{ADDITIONAL INFORMATION}

Supplementary Information accompanies this paper at (https://doi.org/10.1038/ s41386-020-0743-7).

Publisher's note Springer Nature remains neutral with regard to jurisdictional claims in published maps and institutional affiliations.

\section{REFERENCES}

1. Ellman LM, Deicken RF, Vinogradov S, Kremen WS, Poole JH, Kern DM, et al. Structural brain alterations in schizophrenia following fetal exposure to the inflammatory cytokine interleukin-8. Schizophr Res. 2010;121:46-54.

2. Knuesel I, Chicha L, Britschgi M, Schobel SA, Bodmer M, Hellings JA, et al. Maternal immune activation and abnormal brain development across CNS disorders. Nat Rev Neurol. 2014;10:643-60.

3. Tenyi T. Neurodevelopment and schizophrenia: data on minor physical anomalies and structural brain imaging. Neuropsychopharmacol Hung. 2011;13:229-32.

4. Meyer U. Developmental neuroinflammation and schizophrenia. Prog Neuropsychopharmacol Biol Psychiatry. 2013;42:20-34.

5. Estes ML, McAllister AK. Maternal immune activation: Implications for neuropsychiatric disorders. Science 2016;353:772-7.

6. Meyer U. Prenatal poly(i:C) exposure and other developmental immune activation models in rodent systems. Biol Psychiatry. 2014;75:307-15.

7. Reisinger S, Khan D, Kong E, Berger A, Pollak A, Pollak DD. The poly(l:C)-induced maternal immune activation model in preclinical neuropsychiatric drug discovery. Pharm Ther. 2015;149:213-26.

8. Murray C, Griffin EW, O'Loughlin E, Lyons A, Sherwin E, Ahmed S, et al. Interdependent and independent roles of type I interferons and IL- 6 in innate immune, neuroinflammatory and sickness behaviour responses to systemic poly I:C. Brain Behav Immun. 2015;48:274-86.
9. Purves-Tyson TD, Weber-Stadlbauer U, Richetto J, Rothmond DA, Labouesse MA, Polesel $M$, et al. Increased levels of midbrain immune-related transcripts in schizophrenia and in murine offspring after maternal immune activation. Mol Psychiatry. 2019. https://doi.org/10.1038/s41380-019-0434-0.

10. Morgan JT, Chana G, Pardo CA, Achim C, Semendeferi K, Buckwalter J, et al. Microglial activation and increased microglial density observed in the dorsolateral prefrontal cortex in autism. Biol Psychiatry. 2010;68:368-76.

11. Smolders S, Notter T, Smolders SMT, Rigo JM, Brone B. Controversies and prospects about microglia in maternal immune activation models for neurodevelopmental disorders. Brain Behav Immun. 2018;73:51-65.

12. Juckel G, Manitz MP, Brune M, Friebe A, Heneka MT, Wolf RJ. Microglial activation in a neuroinflammational animal model of schizophrenia-a pilot study. Schizophr Res. 2011;131:96-100.

13. Laskaris LE, Di Biase MA, Everall I, Chana G, Christopoulos A, Skafidas E, et al. Microglial activation and progressive brain changes in schizophrenia. Br J Pharm. 2016;173:666-80.

14. Liao B, Zhao W, Beers DR, Henkel JS, Appel SH. Transformation from a neuroprotective to a neurotoxic microglial phenotype in a mouse model of ALS. Exp Neurol. 2012;237:147-52.

15. Monji A, Kato TA, Mizoguchi $Y$, Horikawa $H$, Seki $Y$, Kasai $M$, et al. Neuroinflammation in schizophrenia especially focused on the role of microglia. Prog Neuropsychopharmacol Biol Psychiatry. 2013;42:115-21.

16. Manitz MP, Esslinger M, Wachholz S, Plumper J, Friebe A, Juckel G, et al. The role of microglia during life span in neuropsychiatric disease-an animal study. Schizophr Res. 2013;143:221-2.

17. Esslinger M, Wachholz S, Manitz MP, Plumper J, Sommer R, Juckel G, et al. Schizophrenia associated sensory gating deficits develop after adolescent microglia activation. Brain Behav Immun. 2016;58:99-106.

18. Garay PA, Hsiao EY, Patterson PH, McAllister AK. Maternal immune activation causes age- and region-specific changes in brain cytokines in offspring throughout development. Brain Behav Immun. 2013;31:54-68.

19. Giovanoli S, Weber-Stadlbauer U, Schedlowski M, Meyer U, Engler H. Prenatal immune activation causes hippocampal synaptic deficits in the absence of overt microglia anomalies. Brain Behav Immun. 2016;55:25-38.

20. Paolicelli RC, Ferretti MT. Function and dysfunction of microglia during brain development: consequences for synapses and neural circuits. Front Synaptic Neurosci. 2017;9:9.

21. Kobayashi K, Imagama S, Ohgomori T, Hirano K, Uchimura K, Sakamoto K, et al. Minocycline selectively inhibits M1 polarization of microglia. Cell Death Dis. 2013;4:e525.

22. Zhang $L$, Zheng $H$, Wu $R$, Zhu F, Kosten TR, Zhang $X Y$, et al. Minocycline adjunctive treatment to risperidone for negative symptoms in schizophrenia: Association with pro-inflammatory cytokine levels. Prog Neuropsychopharmacol Biol Psychiatry. 2018;85:69-76.

23. Sellgren CM, Gracias J, Watmuff B, Biag JD, Thanos JM, Whittredge PB, et al. Increased synapse elimination by microglia in schizophrenia patient-derived models of synaptic pruning. Nat Neurosci. 2019;22:374-85.

24. Zhu F, Zheng Y, Liu Y, Zhang X, Zhao J. Minocycline alleviates behavioral deficits and inhibits microglial activation in the offspring of pregnant mice after administration of polyriboinosinic-polyribocytidilic acid. Psychiatry Res. 2014;219:680-6.

25. Fujita $Y$, Ishima $T$, Kunitachi $S$, Hagiwara $H$, Zhang $L$, lyo $M$, et al. Phencyclidineinduced cognitive deficits in mice are improved by subsequent subchronic administration of the antibiotic drug minocycline. Prog Neuropsychopharmacol Biol Psychiatry. 2008;32:336-9.

26. Haynes RL, Folkerth RD, Trachtenberg FL, Volpe J, Kinney HC. Nitrosative stress and inducible nitric oxide synthase expression in periventricular leukomalacia. Acta Neuropathol. 2009;118:391-9.

27. Schampel A, Volovitch O, Koeniger T, Scholz CJ, Jorg S, Linker RA, et al. Nimodipine fosters remyelination in a mouse model of multiple sclerosis and induces microglia-specific apoptosis. Proc Natl Acad Sci USA. 2017;114:E3295-E304.

28. Fenn AM, Hall JC, Gensel JC, Popovich PG, Godbout JP. IL-4 signaling drives a unique arginase+/IL-1beta + microglia phenotype and recruits macrophages to the inflammatory CNS: consequences of age-related deficits in IL-4Ralpha after traumatic spinal cord injury. J Neurosci. 2014;34:8904-17.

29. Greenhalgh AD, David S. Differences in the phagocytic response of microglia and peripheral macrophages after spinal cord injury and its effects on cell death. J Neurosci. 2014;34:6316-22.

30. Cherry JD, Olschowka JA, O'Banion MK. Arginase 1+ microglia reduce Abeta plaque deposition during IL-1beta-dependent neuroinflammation. J Neuroinflammation. 2015;12:203.

31. Toedebusch CM, Snyder JC, Jones MR, Garcia VB, Johnson GC, Villalon EL, et al. Arginase- 1 expressing microglia in close proximity to motor neurons were increased early in disease progression in canine degenerative myelopathy, a model of amyotrophic lateral sclerosis. Mol Cell Neurosci. 2018;88:148-57. 
32. Zhao Q, Xie X, Fan Y, Zhang J, Jiang W, Wu X, et al. Phenotypic dysregulation of microglial activation in young offspring rats with maternal sleep deprivationinduced cognitive impairment. Sci Rep. 2015;5:9513.

33. Yao L, Bhatta A, Xu Z, Chen J, Toque HA, Chen Y, et al. Obesity-induced vascular inflammation involves elevated arginase activity. Am J Physiol Regul Integr Comp Physiol. 2017:313:R560-R71.

34. Bhatta A, Yao L, Xu Z, Toque HA, Chen J, Atawia RT, et al. Obesity-induced vascular dysfunction and arterial stiffening requires endothelial cell arginase 1. Cardiovasc Res. 2017;113:1664-76.

35. Ito HT, Smith SE, Hsiao E, Patterson PH. Maternal immune activation alters nonspatial information processing in the hippocampus of the adult offspring. Brain Behav Immun. 2010;24:930-41.

36. Meyer U, Nyffeler M, Engler A, Urwyler A, Schedlowski M, Knuesel I, et al. The time of prenatal immune challenge determines the specificity of inflammationmediated brain and behavioral pathology. J Neurosci. 2006;26:4752-62.

37. Lu J, He C, He G, Wang X, Xu Y, Wu Y, et al. Structural elucidation of new urinary tamoxifen metabolites by liquid chromatography quadrupole time-of-flight mass spectrometry. J Mass Spectrom. 2014;49:570-8.

38. Yin DM, Chen YJ, Lu YS, Bean JC, Sathyamurthy A, Shen C, et al. Reversal of behavioral deficits and synaptic dysfunction in mice overexpressing neuregulin 1. Neuron. 2013;78:644-57.

39. Xia Y, Qi F, Zou J, Yang J, Yao Z. Influenza vaccination during early pregnancy contributes to neurogenesis and behavioral function in offspring. Brain Behav Immun. 2014;42:212-21.

40. Murray KN, Edye ME, Manca M, Vernon AC, Oladipo JM, Fasolino V, et al. Evolution of a maternal immune activation $(\mathrm{mlA})$ model in rats: Early developmental effects. Brain Behav Immun. 2019;75:48-59.

41. Tao Y, Chen YJ, Shen C, Luo Z, Bates CR, Lee D, et al. Erbin interacts with TARP gamma-2 for surface expression of AMPA receptors in cortical interneurons. Nat Neurosci 2013;16:290-9.

42. Veremeyko T, Starossom SC, Weiner HL, Ponomarev ED. Detection of microRNAs in microglia by real-time PCR in normal CNS and during neuroinflammation. J Vis Exp. 2012:4097.

43. Swerdlow NR, Light GA, Cadenhead KS, Sprock J, Hsieh MH, Braff DL. Startle gating deficits in a large cohort of patients with schizophrenia: relationship to medications, symptoms, neurocognition, and level of function. Arch Gen Psychiatry. 2006;63:1325-35.

44. Corcoran R, Mercer G, Frith CD. Schizophrenia, symptomatology and social inference: investigating "theory of mind" in people with schizophrenia. Schizophr Res. 1995;17:5-13.

45. Coiro $P$, Pollak DD. Sex and gender bias in the experimental neurosciences: the case of the maternal immune activation model. Transl Psychiatry. 2019;9:90.

46. Patrich E, Piontkewitz Y, Peretz A, Weiner I, Attali B. Maturation- and sex-sensitive depression of hippocampal excitatory transmission in a rat schizophrenia model. Brain Behav Immun. 2016;51:240-51.

47. Carlezon WA Jr., Kim W, Missig G, Finger BC, Landino SM, Alexander AJ, et al. Maternal and early postnatal immune activation produce sex-specific effects on autism-like behaviors and neuroimmune function in mice. Sci Rep. 2019;9:16928.

48. Nguyen R, Morrissey MD, Mahadevan V, Cajanding JD, Woodin MA, Yeomans JS, et al. Parvalbumin and GAD65 interneuron inhibition in the ventral hippocampus induces distinct behavioral deficits relevant to schizophrenia. J Neurosci. 2014;34:14948-60.

49. Brown JA, Ramikie TS, Schmidt MJ, Baldi R, Garbett K, Everheart MG, et al. Inhibition of parvalbumin-expressing interneurons results in complex behaviora changes. Mol Psychiatry. 2015;20:1499-507.

50. Sohal VS, Zhang F, Yizhar O, Deisseroth K. Parvalbumin neurons and gamma rhythms enhance cortical circuit performance. Nature 2009:459:698-702.

51. Uhlhaas PJ, Singer W. Abnormal neural oscillations and synchrony in schizophrenia. Nat Rev Neurosci. 2010;11:100-13.

52. El-Ansary A, Al-Ayadhi L. GABAergic/glutamatergic imbalance relative to excessive neuroinflammation in autism spectrum disorders. J Neuroinflammation. 2014;11:189.

53. Kelley MH, Wu WW, Lei J, McLane M, Xie H, Hart KD, et al. Functional changes in hippocampal synaptic signaling in offspring survivors of a mouse model of intrauterine inflammation. J Neuroinflammation. 2017;14:180.

54. Shin Yim Y, Park A, Berrios J, Lafourcade M, Pascual LM, Soares N, et al. Reversing behavioural abnormalities in mice exposed to maternal inflammation. Nature. 2017;549:482-87.

55. Canetta S, Bolkan S, Padilla-Coreano N, Song L, Sahn R, Harrison NL, et al. Maternal immune activation leads to selective functional deficits in offspring parvalbumin interneurons. Mol Psychiatry. 2016;21:956-68.

56. Walton NM, Zhou Y, Kogan JH, Shin R, Webster M, Gross AK, et al. Detection of an immature dentate gyrus feature in human schizophrenia/bipolar patients. Transl Psychiatry. 2012;2:e135.
57. Mattei D, Djodari-Irani A, Hadar R, Pelz A, de Cossio LF, Goetz T, et al. Minocycline rescues decrease in neurogenesis, increase in microglia cytokines and deficits in sensorimotor gating in an animal model of schizophrenia. Brain Behav Immun. 2014;38:175-84.

58. Zhao Q, Wang Q, Wang J, Tang M, Huang S, Peng K, et al. Maternal immune activation-induced PPARgamma-dependent dysfunction of microglia associated with neurogenic impairment and aberrant postnatal behaviors in offspring. Neurobiol Dis 2019;125:1-13.

59. Barron H, Hafizi S, Andreazza AC, Mizrahi R. Neuroinflammation and oxidative stress in psychosis and psychosis risk. Int J Mol Sci. 2017;18:651.

60. Vasistha NA, Pardo-Navarro M, Gasthaus J, Weijers D, Muller MK, Garcia-Gonzalez $D$, et al. Maternal inflammation has a profound effect on cortical interneuron development in a stage and subtype-specific manner. Mol Psychiatry. 2019. https://doi.org/10.1038/s41380-019-0539-5.

61. Bragin A, Jando G, Nadasdy Z, Hetke J, Wise K, Buzsaki G. Gamma $(40-100 \mathrm{~Hz})$ oscillation in the hippocampus of the behaving rat. J Neurosci. 1995;15:47-60.

62. Pernia-Andrade AJ, Jonas P. Theta-gamma-modulated synaptic currents in hippocampal granule cells in vivo define a mechanism for network oscillations. Neuron. 2014;81:140-52.

63. Ikrar T, Guo N, He K, Besnard A, Levinson S, Hill A, et al. Adult neurogenesis modifies excitability of the dentate gyrus. Front Neural Circuits. 2013;7:204.

64. Lacefield CO, Itskov V, Reardon T, Hen R, Gordon JA. Effects of adult-generated granule cells on coordinated network activity in the dentate gyrus. Hippocampus. 2012;22:106-16.

65. Bao H, Asrican B, Li W, Gu B, Wen Z, Lim SA, et al. Long-range GABAergic inputs regulate neural stem cell quiescence and control adult hippocampal neurogenesis. Cell Stem Cell. 2017;21:604-17 e5.

66. Zheng J, Li HL, Tian N, Liu F, Wang L, Yin Y, et al. Interneuron accumulation of phosphorylated tau impairs adult hippocampal neurogenesis by suppressing GABAergic transmission. Cell Stem Cell. 2020;26:462-66.

67. Smith KL, Kassem MS, Clarke DJ, Kuligowski MP, Bedoya-Perez MA, Todd SM, et al Microglial cell hyper-ramification and neuronal dendritic spine loss in the hippocampus and medial prefrontal cortex in a mouse model of PTSD. Brain Behav Immun. 2019;80:889-99.

68. Hellwig S, Brioschi S, Dieni S, Frings L, Masuch A, Blank T, et al. Altered microglia morphology and higher resilience to stress-induced depression-like behavior in CX3CR1-deficient mice. Brain Behav Immun. 2016;55:126-37.

69. Chen Z, Jalabi W, Hu W, Park HJ, Gale JT, Kidd GJ, et al. Microglial displacement of inhibitory synapses provides neuroprotection in the adult brain. Nat Commun. 2014;5:4486.

70. Ni J, Wang $X$, Chen S, Liu H, Wang $Y, X u$ X, et al. MicroRNA let-7c-5p protects against cerebral ischemia injury via mechanisms involving the inhibition of microglia activation. Brain Behav Immun. 2015;49:75-85.

71. Xu Y, Xu Y, Wang $Y$, Wang $Y$, He L, Jiang $Z$, et al. Telmisartan prevention of LPSinduced microglia activation involves M2 microglia polarization via CaMKKbetadependent AMPK activation. Brain Behav Immun. 2015;50:298-313.

72. Zhang J, Xie X, Tang $M$, Zhang J, Zhang B, Zhao $Q$, et al. Salvianolic acid B promotes microglial M2-polarization and rescues neurogenesis in stress-exposed mice. Brain Behav Immun. 2017;66:111-24.

73. Kiyofuji K, Kurauchi Y, Hisatsune A, Seki T, Mishima S, Katsuki H. A natural compound macelignan protects midbrain dopaminergic neurons from inflammatory degeneration via microglial arginase-1 expression. Eur J Pharm. 2015;760:129-35.

74. Fouda $A Y, X u Z$, Shosha E, Lemtalsi T, Chen J, Toque HA, et al. Arginase 1 promotes retinal neurovascular protection from ischemia through suppression of macrophage inflammatory responses. Cell Death Dis. 2018;9:1001.

75. Lee S, Latha K, Manyam G, Yang Y, Rao A, Rao G. Role of CX3CR1 Signaling in malignant transformation of gliomas. Neuro Oncol. 2020. https://doi.org/10.1093/ neuonc/noaa075.

76. Ridderstad Wollberg A, Ericsson-Dahlstrand A, Jureus A, Ekerot $P$, Simon S, Nilsson $M$, et al. Pharmacological inhibition of the chemokine receptor CX3CR1 attenuates disease in a chronic-relapsing rat model for multiple sclerosis. Proc Natl Acad Sci USA. 2014;111:5409-14.

77. Lehmann ML, Cooper HA, Maric D, Herkenham M. Social defeat induces depressive-like states and microglial activation without involvement of peripheral macrophages. J Neuroinflammation. 2016;13:224.

78. Amaral DG, Scharfman HE, Lavenex P. The dentate gyrus: fundamental neuroanatomical organization (dentate gyrus for dummies). Prog Brain Res. 2007;163: 3-22.

79. Kheirbek MA, Drew LJ, Burghardt NS, Costantini DO, Tannenholz L, Ahmari SE, et al. Differential control of learning and anxiety along the dorsoventral axis of the dentate gyrus. Neuron 2013;77:955-68.

80. Deng W, Saxe MD, Gallina IS, Gage FH. Adult-born hippocampal dentate granule cells undergoing maturation modulate learning and memory in the brain. J Neurosci. 2009;29:13532-42. 
Modulating microglia activation prevents maternal immune activation...

Y Xia et al.

1908

81. Guo N, Yoshizaki K, Kimura R, Suto F, Yanagawa Y, Osumi N. A sensitive period for GABAergic interneurons in the dentate gyrus in modulating sensorimotor gating. J Neurosci. 2013;33:6691-704.

82. Espinoza C, Guzman SJ, Zhang X, Jonas P. Parvalbumin(+) interneurons obey unique connectivity rules and establish a powerful lateral-inhibition microcircuit in dentate gyrus. Nat Commun 2018;9:4605.

83. Sambandan S, Sauer JF, Vida I, Bartos M. Associative plasticity at excitatory synapses facilitates recruitment of fast-spiking interneurons in the dentate gyrus. J Neurosci 2010;30:11826-37.

84. Leutgeb JK, Leutgeb S, Moser MB, Moser El. Pattern separation in the dentate gyrus and CA3 of the hippocampus. Science 2007;315:961-6.
85. Giovanoli S, Engler H, Engler A, Richetto J, Feldon J, Riva MA, et al. Preventive effects of minocycline in a neurodevelopmental two-hit model with relevance to schizophrenia. Transl Psychiatry. 2016;6:e772.

86. Lima-Silveira L, Accorsi-Mendonca D, Bonagamba LGH, Almado CEL, da Silva MP, Nedoboy PE, et al. Enhancement of excitatory transmission in NTS neurons projecting to ventral medulla of rats exposed to sustained hypoxia is blunted by minocycline. J Physiol. 2019;597:2903-23.

87. Sorge RE, Mapplebeck JC, Rosen S, Beggs S, Taves S, Alexander JK, et al. Different immune cells mediate mechanical pain hypersensitivity in male and female mice. Nat Neurosci. 2015;18:1081-3. 